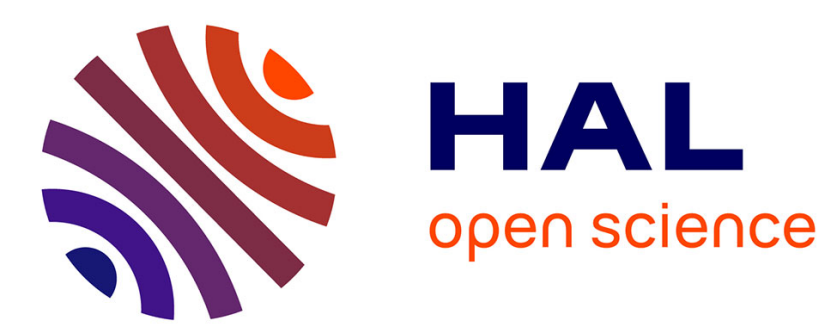

\title{
A constructive method for deriving finite elements of nodal type
}

\author{
Jean-Pierre Hennart, Jérôme Jaffré, Jean Roberts
}

\section{To cite this version:}

Jean-Pierre Hennart, Jérôme Jaffré, Jean Roberts. A constructive method for deriving finite elements of nodal type. [Research Report] RR-0541, INRIA. 1986. inria-00076013

\section{HAL Id: inria-00076013 https://hal.inria.fr/inria-00076013}

Submitted on 24 May 2006

HAL is a multi-disciplinary open access archive for the deposit and dissemination of scientific research documents, whether they are published or not. The documents may come from teaching and research institutions in France or abroad, or from public or private research centers.
L'archive ouverte pluridisciplinaire HAL, est destinée au dépôt et à la diffusion de documents scientifiques de niveau recherche, publiés ou non, émanant des établissements d'enseignement et de recherche français ou étrangers, des laboratoires publics ou privés. 


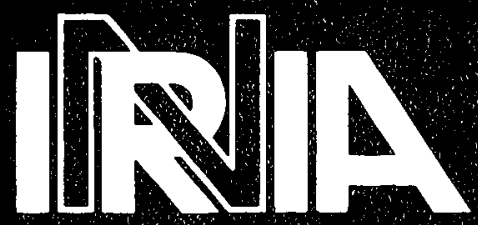

CENTRE DE ROCQUENCOURT

\section{Rapports de Recherche}

\section{A CONSTRUCTIVE METHOD FOR DERIVING FINITE ELEMENTS OF NODAL TYPE}

Instutut National de Recherche en. et en Automatique

Domaina de Volvecau

Roogencourt , 3 ,

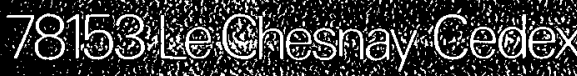

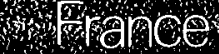

\section{Jean - Pierre HENNART Jérôme JAFFRE Jean Elizabeth ROBERTS}




\title{
A CONSTRUCTIVE METHOD FOR DERIVING
}

EINITE ELEMENTS OF NODAL TYPE

\author{
Jean-Plerre HENNART * \\ Jếôme JAFFRE \\ and Jean Elizabeth ROBERTS
}

* IIMAS-Unam A partado Postal 20-726, 01000 México, D.F. (MEXIQUE)

* IN RIA Domaine de Voluceau-Rocquencourt BP. 10578153 Le Chesnay, Cédex (France). 


\section{RESUME}

Dans cet article, on propose un algorithıne de construction de méthodes nodales correspondant à divers éléments finis, non conformes, mixtes, bidimensionnels ou tridimensionnels. On montre qu'on peut ainsi obtenir plusieurs méthodes classiques aussi bien que certaines plus récentes, et que cet algorithme produit une démonstration simple de l'unisolvence pour ces inéthodes. Finalement on utilise cet algorithme pour obtenir une méthode nodale tridimensionnelle de type BDM.

\section{A B S TRACT}

In this paper, we propose an algorithm to derive nodal methods corresponding to various two and three-dimensional non conforming and mixed finite elements. We show that this algorithm can be used to obtain several classical schernes as well as some more recently developed schemes, and that it leads to a sinple proof of unisolvence for these methods. Finally we use our inethod to obtain a three dimensional nodal scheme of BDM type. 


\section{I - INTRODUCTION}

Nodal methods are methods which try to combine the most favorable features of the finite element method (f.e.m.) and the finite difference method (f.d.m.), two discretization methods which, a priori, are quite different. From the f.e.m., they borrow a piecewise continuous, usually polynomial, approximation to the solution of the problem considered. Like the f.d.m., they usually produce quite regular and well structured algebraic systems of equations : they are in fact fast solvers, well suited for parallel processing. This is true of course for relatively regular meshes, the most typical one being probably the "union-of-rectangles" mesh.

Nodal methods were introduced in nuclear engineering during the late 1970's: first references are Refs. $[12,19,20,26]$ while Refs. $[9,13,28]$ are essentially review papers where many more references can be found. In reactor analysis, there have been applloations in static diffusion and in space-time dynamics, as well as. in transport, covering, by the way the three classical types of partial differential equations elliptic, parabolic and hyperbolic, all of them linear or quasi-linear. In reactor calculations, the sequence is almost always the following : a preprocessing operation is first performed whereby fine details of the reactor core are homogenized into a fairly coarse and regular array of homogeneous assemblies, a coarse-mesh or nodal calculation is then performed and if fine details of neutron flux are wanted, a postprocessing follows, ylelding the local maxima of the flux where hot spots can be expected.

It is very interesting to note that in many fields of application, which have nothing to do with reactor calculation, we are confronted with very similar situations, except that the pre and/or postprocessing operations are usually absent, in other words, situations for which everything boils down to the coarse-mesh or nodal calculation. This is typically the case for the numerical simulation of flow in porous media. Traditionally, finite differences are used here over fairly regular meshes of the union-of-rectangles type. This is so because in most cases the boundary of the region to be modelled is not very well-known, so that a regular grid is fairly natural. Moreover, the coefficlents of the modelled equations, such as the porosity of the medium, its permeability, 
etc., are simply not known with enough accuracy to allow for more than a mean or average value per coarse cell. This is for instance the case in underground water hydrology, oil reservoir simulation, nuclear waste disposal, etc., applications, where the people involved basically speak "finite differences". Oitside of the porous medium field, we could mention similar applications, for instance, those in atmospheric pollution modeling.

In refs. $[11,17,18]$ the relationship existing between some early nodal schemes and nonconforming formulations of the f.e.m. was made explicit : that these early nodal schemes do not climb correctly in order was shown [11], a family of nodal schemes which does wis proposed [17], and "numerical evidence" to support that claim was exhibited [18]. By a "family of nodal schemes climbing correctly in order", we mean a family such that if $k$ is a nonnegative integer index attached to each member of the family, convergence orders in the $\mathrm{L}^{2}$ norm of $O\left(\mathrm{~h}^{\mathrm{k}+2}\right)$ can be expected for the error between the approximation $\mathrm{u}_{\mathrm{h}}$ and the solution $u$, where $h$ is a measure of the size of the cells in the mesh. More recently the relationship existing between this new family of nodal schemes and extensions of mixed-hybrid finite elements [1] was demonstrated [16]. At present, mixed and mixed-hybrid finite elements are particularly fashionable in research codes for oll reservoir simulation $[5,6,10]$. It is therefore interesting to establish the connection between schemes used in two fields of application apparently so far away, showing incidentally that our above remarks were well founded. Applications to neutron diffusion and transport have also been presented $[8,15]$.

A characteristic of nodal schemes leading to reduced couplings is that the "nodes", "cells", "blocks", or "elements" are basically coupled by interface variables which usually are moments of the unknown function or sometimes its values at Gauss points. To these variables, we must add some interior variables, in principle moments of the unknown function over the cell. These edge and cell moments are linear functionals which will hopefully capture the essential behavior of the unknown function. This leads us to the concept of finite elements of nodal type, for which the basic parameters or degrees of freedom are edge and cell moments. In rectangular $\mathbb{R}^{n}$ geometry, which is the only geometry we shall consider in the following, it is always possible to refer to the referenco cell $[-1,+1]^{n}$ by a simple diagonal affine mapping; thus, we shall define the 
edge and cell moments with respect to the reference cell. In 2D for instance, let $\mathrm{u}(\mathrm{x}, \mathrm{y})$ be the unknown function. Its edge moments will be defined by :

and

$$
\begin{aligned}
& m_{1}^{1}(u) \equiv \int_{-1}^{+1} P_{i}(y) u(-1, y) d y / N_{i}, \\
& m_{R}^{1}(u) \equiv \int_{-1}^{+1} P_{i}(y) u(+1, y) d y / N_{1}, \\
& m_{D}^{i}(u) \equiv \int_{-1}^{+1} P_{i}(x) u(x,-1) d x / N_{i},
\end{aligned},
$$

$$
m_{U}^{i}(u) \equiv \int_{-1}^{+1} P_{i}(x) u(x,+1) d x / N_{i} \text {, }
$$

where $L, R, D$, and $U$ stand for "left", "right", "down" and "up" respectively, $P_{1}$ is the normalized Legendre polynomial of degree $i$ over $[-1,+1]$, and $N_{i}=2 /(21+1)$ is a convenient normalization factor. Cell moments of $u$ are defined by

$$
\cdot m_{C}^{i j}(u) \equiv \int_{C} P_{i}(x) P_{j}(y) u(x, y) d x d y / N_{i} \cdot N_{j} \cdot
$$

In $3 D$, we shall have to consider face moments instead of edge moments, $m_{L}^{i j}, m_{R}^{i j}, m_{D}^{i j}, m_{U}^{1 j}, m_{F}^{1 j}$, and $m_{B}^{i j}$, where $F$ and $B$ correspond to "front" and "back". Similarly the cell moments will then become $\mathrm{m}_{C}^{i j k}$.

Such finite elements are clearly non conforming in $H^{2}(\Omega)$ as elements $u_{h}$ of the approximation space are continuous only in the mean, plus possibly in some of their higher moments, through the interfaces. If a primal f.e.m. is considered, the approximation space must pass the "patch test" [27] to some order : for an $L^{2}$ convergence of order $O\left(h^{k+2}\right), k \in \mathbb{N}=\{0,1,2 \ldots\}$, it is sufficient $[7,14]$ that

(i) $\mathscr{P}_{k+1}$ be included in the space of polynomials describing $u_{h}$ witilin the cell, where

$$
\mathscr{Q}_{k} \equiv\left\{x^{a} y^{b} ; 0 \leq a+b \leq k\right\}
$$

(ii) elements of the approximation space have $k+1$ moments continuous through each edge. See Appendix $C$ for more details. 
In the following other polynomial spaces will be considered such as

$$
Q_{k, \ell} \equiv\left\{x^{a} y^{b} ; 0 \leqq a \leq k, 0 \leqq b \leqq \ell\right\}
$$

and $Q_{k} \equiv Q_{k, k^{*}}$ In 3D, a trivial modification would lead us to $\mathscr{Q}_{k, l, m}$ with again $\mathscr{Q}_{k} \equiv \mathscr{Q}_{k, k, k}$

The general issue of unisolvence can be stated as follows : given a finite element on the cell C (classical or of nodal type), a set of degrees of freedom $D$ is defined as well as a space $S$ of polynomials which is the space of restrictions to the cell $c$ of functions $u_{h}$ in the approximation space. In classical finite elements, the degrees of freedom usually are values and possioly derivatives of the functions $u_{h}$ in $s$ at special nodes of the given element $C$, Including in principle its vertices (which by the way leads to the strong couplings typical of the classical f.e.m.). With finite elements of nodal type, these degrees of freedom are edge moments $m_{E}^{i}\left(u_{h}\right)$, where $E$ stands for $L$, $R$, $U$, or $D$, and cell moments $m_{C}^{1 j}\left(u_{h}\right)$. To say that $S$ is unisolvent with respect to $D$ means that any member of $S$ is uniquely determined in terms of the 1 inear functionals in D. A necessary condition for unisolvence is, as we recall, $\operatorname{dim}(S)=\operatorname{card}(D)$ but it is not sufficient. The two basic techniques for proving unisolvence are

(i) to exhibit dual basis functions, $i$.e. basis functions belonging to $S$ of the $\delta_{i j}$ type ("the $i^{\text {th }}$ functional of $D$ is one for the $i^{\text {th }}$ basis function and zero for the other basis functions"); then, as $\operatorname{dim}(S)=\operatorname{card}(D)$, if there is a solution it is unique, or

(ii) to prove that if all the functionals in D are zero for a function in $S$, then that function must be identical to zero.

Our objective in this paper is to consider a variety of situations of the nodal type where some edge and cell moments are specified, i.e. D is given, while $S$ is not known a priori. The edge and cell moments often have some physical meaning so it is quite natural to give them beforehand : the space $s$ of basis functions is then to be determined in a way that leads to unisolvonue. Given $D$, there are many possible choices of the space $S$ and our concern hero is 
to determine in a constructive way an $S$ which has the right approximation properties, not only for the inner representation in a cell $\left(\mathscr{P}_{k+1} \subset S\right)$ but also for the coupling betwen neighboring cells (continuity of $(k+1)$ edge moments).

In section 2, a general constructive algorithm for such finite elements of the nodal type in $2 \mathrm{D}$ is proposed. It is applied in section 3 to well known situations where it is shown to reproduce previous bases. In section 4, we apply it to derive the (new) nodal extensions of the recent Brezzi-Douglas-Marini (BDM) mixed finite elements [4]. Section 5 is devoted to 3D situations with examples given in section 6. Two appendices finally. give examples of basis functions in 2 and $3 D$, while a third one gives a general theorem leading to error estimates for nodal finite elements. 


\section{II - THE TWO-DIMENSIONAL CASE}

Before presenting our constructive algorithm in $2 \mathrm{D}$, we would like to introduce some notation which will be useful, even later in 3D situations. As we said before, $P_{1}$ will be the nor tallzed Legendre polynomial of degree $i$ over $[-1,+1]$ with the well known properiles

and

$$
\begin{aligned}
& P_{i}(+1)=1, \\
& P_{1}(-1)=(-1)^{1}, \\
& \int_{-1}^{+1} P_{i}(x) P_{j}(x) d x=\delta_{1 j} N_{i},
\end{aligned}
$$

$$
P_{i}(-x)=(-1)^{i} P_{i}(x) \text {. }
$$

Let us introduce moreover

and

$$
P_{1 j}(x, y) \equiv P_{1}(x) P_{j}(y)
$$

$$
P_{i j k}(x, y, z) \equiv P_{1}(x) P_{j}(y) P_{k}(z) \text {. }
$$

For a given set $D$ of degrees of freedom for functions defined on the reference cell $C=[-1,+1]^{2}$, our algorithm will define the space $S$ of polynomials, in which the restriction to $C$ of the unknown function is to be approximated, by specifying a basis of normalized Legendre polynomials. The choice of the basis of polynomials $P_{a b}$ instead of the more usual basis of monomials $x^{a} y^{b}$, with the normalizations we adopt, ylelds nice simplifications not only in the unisolvence proofs but also in the calculation of the dual basis functions in $S$.

In 2D, a convenient way to describe the spaces $S$ which will be chosen for a given element type (elther classical or nodal) is with a Pascal triangle in which the different polynomials $P_{a b}$ forming the basis of $S$ are presented in $a$ systematic way. In fig. 1, this representation is sketched for the classioal choices of spaces $S$ for triangles and for rectangles. In all of the ficires, ab is used to denote $P_{a b}$. 
To set the scene for our algorithm, we shall make the following assumptions, which are not really restrictive and can in fact be dropped in special applications : we shall first assume that on the left and right (or vertical) edges of a given cell, momeits of order 0 through $k$ are given while on the up and down (or horizontal) edgus, moments of order 0 through \& are given. Here $k$ and $\ell$ are integers $\geq-1$, a value of -1 indicating that no moments are specified on the corresponding edges. Further we assume that, within the cell, moments with respect to $\mathscr{P}_{m}, \mathbb{Q}_{m, n}$ or $\mathscr{Q}_{m, n} \cup \mathscr{Q}_{n, m}$ are prescribed. Again $m$ is an integer $\geq-1$, while $n$ when it is specified, is a nonnegative integer, a value of -1 for $m$ indicating that no cell moments are prescribed. Clearly, one at least of $k, \ell$, and $m$ should be nonnegative.

In the figures representing the degrees of freedom $D$, the edge moments will be represented by small line segments perpendicular to the corresponding edge, the absence of such segments meaning that the corresponding index $k$ or 2 is negative. The presence of $\mathscr{Q}_{m}, \mathscr{Q}_{m, n}$ or $\mathscr{Q}_{m, n} \cup \mathscr{Q}_{n, m}, m, n \in \mathbb{N}$, within the cell will mean that the corresponding interior or cell moments are prescribed, while the absence of any symbol implies that no cell moments are present. This representation should becone clear with the proposed examples.

In 2D, our algorithm can now be stated as follows :

\section{ALGORITHM 1}

(i) All the polynomials $P_{1 j}$ belonging to the specified set $\mathscr{P}_{m^{\prime}} \mathscr{Q}_{m, n}$ or $Q_{m, n} \cup Q_{n, m}$ must be first included in the basis for $S$. In the Pascal triangle representation, these polynomials will correspond to the $1 j$ positions and we shall say that they constitute the "trunk" of the basis.

(1i) Outside of the area occupied in the Pascal triangle by the trunk, $k$ "two-boned legs" must be pulled out to the left and $\ell$ "two-boned legs" to the right, a "bone" corresponding to a position in the triangle.

More precisely, the $2(k+1)$ vertical edge moments and the $2(\ell+1)$ horizontal edge moments give $r$ ise to the basis elements $P_{a_{i}, i}$ and $P_{a_{i}}+1, i$, $i=0, \ldots, k$, and $P_{j, b}$ and $P_{j, b_{j}+1}, j=0, \ldots, l$, where the $a_{i}{ }^{\prime} s$ and $b_{j}^{\prime} s$ 
indicating the points of departure of the left legs and right legs respectively, are determined as follows :

For $0 \leq 1 \leq \min \{k, l\}, a_{1}$ is the least integer $a, a \geq 1$, such that $p_{a, i}$ is not included in the trunk. Simi $a r l y, b_{i}$ is the least integer $b, b \geq i$, such that $P_{i, b}$ is not ir luded in the trunk. Note that for $0 \leqq i, j \leq \min \{k, l\}$ the $i^{\text {th }}$ left $l e g$ and the $j^{\text {th }}$ right leg can overlap only in case $i=j$ and the point of departure of the legs is the same, i.e. $a_{i}=b_{j}=i=j$.

(iii) If it happens that the point of departure of two legs corresponding to the $i^{\text {th }}$ vertical and the $i^{\text {th }}$ horizontal moments, is common to both legs, these two legs will now be attached to the corresponding polynomial $P_{i 1}$ ' and we shall include in the basis, in addition to $P_{1 i}, P_{i+1,1}$, and $P_{1, i+1}$, the element $P_{i+2, i^{-}} P_{i, i+2^{*}}$ We shall say that $P_{i+2, i}$ and $P_{i, i+2}$ are also bones of the $i^{\text {th }}$ left and $1^{\text {th }}$ right legs, respectively, and that $P_{i, i}$ is the "i-pelvis".

(iv) If $k \neq l$, say $k>\ell$, the $i^{\text {th }}$ left legs, $i=\ell+1, \ldots, k$, or rather their points of departure $a_{1}$, still have to be defined. In this case, we permit $a_{i}$ to be less than $i$ in order to fill in the Pascal triangle as much as possible from the top but $a_{i}$ still should be chosen so that the $i^{\text {th }}$ left leg does not meet one of the right legs and does not lie under one of them. Thus for $\ell+1 \leq i \leq k, a_{i}$ is the least integer $a, a \geq \ell+1$, such that $P_{a i}$ is not included in the trunk.

Then we have :

\section{LEMMA 1}

Given a set $D$ of degrees of freedom conforming to the stated hypotheses, the space $S$ constructed by the above algorithm is unisolvent with respect to) $D$. 


\section{PROOF}

Let us first show that $\operatorname{dim}(S)=\operatorname{card}(D)$. There are clearly as many elements in the trunk as cell moments. The edge moments by hypothesis come by pairs (vertical and horizontal ones): to each pair corresponds a two-boned leg in the separated case, while in the nonseparated case we have for two intersecting pairs (say the $i^{\text {th }}$ horizontal and vertical moments) a pelvis plus two two-boned legs or five elements of wich four only are independent, so that clearly $\operatorname{dim}(S)=\operatorname{card}(D)$.

Let us now show unisolvence. Assume that all the given moments are zero. Any member $u_{h}$ of $s$ is of the form

$$
u_{h}=\sum_{a b} C_{a b} P_{a b}
$$

where $a b$ is any allowed position in the Pascal triangle. Clearly in the case of joined legs, $C_{1,1+2}=-C_{1+2,1}$. We shall show that all the coefficients $C_{a b}$ in (7) are zero.

First, for all the cell moments, using (2), (5) and (7), we have

$$
c_{1 j}=m_{C}^{1 j}\left(u_{n}\right)=0
$$

where ij is any allowed position in the trunk.

Consider any pair, say the $i^{\text {th }}$, of vertical moments with $0 \leqq 1 \leqq \min \{k, l\}$, not attached to a right leg. From (1), (5), (7), and (8), we obtain

and

$$
c_{a i} P_{a}(-1)+c_{a+1, i} P_{a+1}(-1)=m_{L}^{1}\left(u_{n}\right)=0
$$

$$
C_{a 1} P_{a}(+1)+C_{a+1,1} P_{a+1}(+1)=m_{R}^{i}\left(u_{h}\right)=0
$$

where ai and $a+1, i$ are the two positions in the Pascal triangle outside of the trunk corresponding to the $i^{\text {th }}$ left leg, assumed to be alone. Using (5), (9) becomes

$$
c_{a i}-c_{a+1, i}=c_{a i}+c_{a+1, i}=0 \text {, }
$$


implying that both $C_{a 1}$ and $C_{a+1,1}$ are zero. The same is true for all the solitary right legs corresponding to $i^{\text {th }}$ horizontal moments for $0 \leqq i \leqq m i n\{k, l\}$.

Let us now look at the case of joined legs with pelvis at i1. The equations for $m_{L}^{1}, m_{R}^{1}, m_{D}^{i}$, and $m_{U}^{i}$ together with (8) and (10) yield.

$$
\begin{aligned}
& c_{11}-c_{1+1,1}+c_{1+2,1}=0 \\
& c_{11}+c_{1+1,1}+c_{1+2,1}=0 \\
& c_{11}-c_{1,1+1}+c_{1,1+2}=0
\end{aligned}
$$

and

$$
c_{i 1}+c_{1,1+1}+c_{1, i+2}=0 \text {. }
$$

so tinat clearly $C_{i+1,1}=c_{1,1+1}=0$. We are left with

and

$$
c_{11}+c_{1+2,1}=0
$$

$$
c_{11}+c_{1,1+2}=0
$$

implying

$$
c_{11}=c_{1,1+2}=c_{1+2,1}=0 \text {, }
$$

since $c_{1,1+2}=-c_{1+2,1}$

Finally we consider a pair of $i^{\text {th }}$ edge moments for $i>\min \{k, \ell\}$, say $k>\ell$, so that $\ell+1 \leq i \leq k$. From (1), (5), and (7) we have :

and

$$
m_{L}^{1}\left(u_{n}\right)=\sum_{j} c_{j 1} P_{j}(-1) \text {. }
$$

$$
m_{R}^{i}\left(u_{n}\right)=\sum_{j} c_{j 1} P_{j}(+1) \text {. }
$$

However from (8), (10), and (11), we deduce that $c_{i j}=0$ for $j<$ a where $P_{a j}$ and $P_{a+1, i}$ are the two bones of the $i^{\text {th }}$ leg. Thus we obtain the equations $(9)$ and (i0) for $\bar{x}+i \leq i \leq k$ and consequentiy that $c_{a}$ and $c_{a+1, i}$ are zero. QED. 
Remark 1 It should be clear from the above demonstration that when edge moments are present there are infiniteiy many spaces $s$ that could be chosen, $D$ being fixed. Given the cell moments, the trunk is fixed once and for all, but the legs could have holes provided their bones retained the correct parities. The $1^{\text {th }}$ left leg for instance could perfectly well combine $P_{a i}$ and $P_{a+3, i}$ instead of $P_{a 1}$ and $P_{a+1,1}$. However since we want to fill in the Pascal trlangle froin the top, our cholce is the most natural one.

Reark 2 Our choice of normalization (1) and (2) leads to particularly eleyant equations. Looking at the left hand sides of (8), (10) and (11). we see that all the coefficients take as values $-1,0$ or +1 . In the determination of the dual basis functions corresponding to each of the moments of $D$, the second members have zero components in all positions, as above, except one of them where the value is one. In simple situations, i.e. when $\operatorname{dim}(S)=N$ is not too high, these equations can usually be solved by simple inspection. For more complex cases, the computer could be used.

Renark 3 With our anatomical analogles, our nodal finite elements are clearly potentlal monsters, with or without a trunk, several legs on each side, and eventually one or more pelvises. 


\section{III - SOME EXAMPLES}

Example 1: The Langenbuch-Maurer-Werner $\Sigma$ or sum nodal schemes $[19,20]$.

For this example :

$$
\begin{aligned}
& D_{k}=\left\{m_{L}^{0}\left(u_{h}\right), m_{R}^{0}\left(u_{h}\right), m_{U}^{0}\left(u_{h}\right), m_{D}^{0}\left(u_{h}\right),\right. \\
& m_{C}^{10}\left(u_{h}\right), \quad i=0, \ldots, k, \\
&\left.m_{C}^{0 j}\left(u_{h}\right), \quad j=1, \ldots, k\right\}, \operatorname{card}\left(D_{k}\right)=2 k+5, \\
& s_{k}= Q_{k+2,0} \cup Q_{0, k+2}, \\
& \forall k \in \mathbb{N}
\end{aligned}
$$

These schemes are illustrated in Fig. 2. Clearly only zero ${ }^{\text {th }}$ order edge moments will be continuous while $\mathscr{P}_{1} \subset s_{k}$ and $\mathscr{P}_{2} \not s_{k} \forall k$. Correspondingly, these nodal schemes do not climb correctly in order. As shown earlier [11], they exhibit $L^{2}$ convergence of $O\left(h^{2}\right) \forall k$.

Example 2. The Hennart nodal schemes $[17,18]$.

These nodal scheme have been built from the nodal schemes of Example 1, with the idea of foroing them to climb correctly in order.

$$
\begin{aligned}
D_{k}= & \left\{m_{L}^{1}\left(u_{h}\right), m_{R}^{1}\left(u_{h}\right), m_{D}^{1}\left(u_{H}\right), m_{U}^{1}\left(u_{H}\right), i=0, \ldots, k,\right. \\
& \left.m_{C}^{i j}\left(u_{h}\right), 1, j=0, \ldots, k\right\}, \operatorname{card}\left(D_{k}\right)=(k+1)(k+5), \\
S_{k}= & \mathscr{Q}_{k+2, k} \cup \mathscr{Q}_{k, k+2}, \operatorname{dim}\left(S_{k}\right)=(k+1)(k+5), \\
& \forall k \in \mathbb{N}
\end{aligned}
$$


Dual basis functions are given in Ref.[17]. These schemes are illustrated in Fl 8. 3. Clearly, they climb correctly in order.

Example 3. The Raviart-Thomas mixed finite elements [25].

In the mixed formulation of the f.e.m., a second order elifptic problem like$$
-\Delta u=f, \quad \text { in } \Omega
$$

is written as a couple of first order equations

$$
\begin{aligned}
& \vec{v}+\vec{\nabla} u=0 \\
& \vec{\nabla} \cdot \vec{v}=f \quad, \text { in } \Omega
\end{aligned}
$$

A weak form of (15) is then obtained by multiplication by test functions and integration by parts. As a result $\vec{v}$ and $u$ are looked for (independently) in $H(d i v ; \Omega)$ and $L^{2}(\Omega)$, respectively, the Raviart-Thomas mixed finite elements constituting a conforming finite element approximation of $H(d i v ; \Omega) \times L^{2}(\Omega)$ (climbing correctly in order).

For the scalar variable u

$$
\begin{gathered}
D_{k}=\left\{m_{C}^{1 j}\left(u_{h}\right), \quad 1, j=0, \ldots, k\right\}, \operatorname{card}\left(D_{k}\right)=(k+1)^{2}, \\
S_{k}=Q_{k}, \operatorname{dim}\left(S_{k}\right)=(k+1)^{2}, \\
\forall k \in \mathbb{N},
\end{gathered}
$$

as shown in Fig. 4.

$$
\begin{aligned}
& \text { For the vector varlable } \vec{v}=\left(v_{1}, v_{2}\right) \text {, we shall have } \\
& D_{k}=D_{k 1} \cup D_{k 2}, S_{k}=S_{k 1} \times S_{k 2}
\end{aligned}
$$

with 


$$
\begin{aligned}
& D_{k 1}=\mid m_{H}^{i}\left(v_{n 1}\right), \quad 1=0, \ldots, k, \\
& \left.m_{C}^{1 j}\left(v_{h 1}\right), 1=0, \ldots, k-1, j=0, \ldots, k\right\} \\
& D_{k 2}=1 m_{v}^{1}\left(v_{h 2}\right), \quad 1=0, \ldots, k, \\
& \left.m_{c}^{1 j}\left(v_{h 2}\right), i=0, \ldots, k \quad, j=0, \ldots, k-1\right\} \\
& \operatorname{card} D_{k}=2(k+1)(k+2) \\
& s_{k 1}=Q_{k+1, k}, S_{k 2}=\mathscr{Q}_{k, k+1}, \quad \operatorname{dim} s_{k}=2(k+1)(k+2), \\
& \forall k \in \mathbf{N}
\end{aligned}
$$

where $m_{H}^{1}$ (resp. $m_{v}^{1}$ ) stands for $m_{L}^{1}$ and $m_{R}^{1}$ (resp. $m_{D}^{1}$ and $m_{U}^{1}$ ). For $u_{h}$ as well as for $\vec{v}_{h}$, dual basis functions have been given [16]. In the same reference, the nodial schemes of Example 2 were shown to be extensions a la Arnold-Brezz1 [1] of the Raviart-Thomas mixed finite elements, under appropriate hypotheses. These mixed fintte elements have been extended by Nédélec to 3D [23]. Accordingly, we shall call the nodal schemes of example 2 modal finite elenents of the RTN type. Fig.5 illustrates (17) for the first component $v_{1}$ of $\vec{v}$.

Before moving to example 4, we would like to offer some comments. In all the previous examples, as we have seen, our algorithm gave us exactly the spaces $S$ (or $S_{k}$ ) which have been obtained independently by quite different approaches $[17,19,20,25]$. This confirms a posteriori its validity. The fact. that tine corresponding elements (here in $\mathrm{H}^{1}(\Omega)$ or $\mathrm{H}(\mathrm{div} ; \Omega)$ ) do or do not olimb correctly in order is then derived by direct inspection of $S$ and $D$ each oase. In example 4, as in the previous example, we are concerned with a space of scalars and a space of vectors. However, in this example, the two components of the vectors are not independently determined. Thus our algorithm and proof of unisolvence cannot be directly applied, but we would like to show how our general appropech to unisolivence can be explibteid to provide a clean and direct demonstration. 
Example 4: The Brezzi-Douglas-Marini mixed finite elements [4].

These recent mixed finite elements are conforming in $H(d i v ; \Omega) \times L^{2}(\Omega)$, like the RTN mixed finite elements. The basic idea behind their development was to achieve the same convergence orders as with their RTN counterparts but with (asymptotically) only fifty percent of the unknowns. Assuming that $k \in \mathbb{N}_{*}$ (i.e. $k \geq 1$ ), the scalar variable is taken to be in $\mathscr{P}_{k-1}$ instead of $Q_{k}$ ' namely

$$
\begin{aligned}
& D_{k}=\left\{m_{C}^{1 j}\left(u_{n}\right) \quad, \quad 0 \leq i+j \leq k-1\right\}, \operatorname{card}\left(D_{k}\right)=k(k+1) / 2 \text {, } \\
& s_{k}=\mathscr{P}_{k-1} \quad, \operatorname{dim}\left(s_{k}\right)=k(k+1) / 2 \text {, } \\
& \forall k \in \mathbb{N}_{*}
\end{aligned}
$$

as shown in Fig. 5 .

$$
\begin{aligned}
& \text { For the vector variable } \vec{v}=\left(v_{1}, v_{2}\right) \\
& D_{k}=\left\{m_{H}^{i}\left(v_{n 1}\right), \quad, i=0, \ldots, k,\right. \\
& m_{C}^{i j}\left(v_{h 1}\right), \quad 0 \leq i+j \leq k-2, \\
& m_{v}^{1}\left(v_{n 2}\right), \ldots, 1=0, \ldots, k, \\
& \left.m_{C}^{i j}\left(v_{n 2}\right), \quad 0 \leqslant i+j \leqq k-2\right\} \\
& \operatorname{card} D_{k}=k^{2}+3 k+4 \text {. } \\
& s_{k}=\left\{\mathscr{P}_{k} \times \mathscr{P}_{k}\right\} \oplus \operatorname{span}\left\{\left(\begin{array}{c}
P_{k+1,0} \\
-(2 k+1) P_{k}
\end{array}\right),\left(\begin{array}{c}
-(2 k+1) P_{1 k} \\
P_{0, k+1}
\end{array}\right)\right\} \\
& \operatorname{dim} S_{k}=k^{2}+3 k+4, \\
& \forall k \in \mathbb{N}_{*} \text {, }
\end{aligned}
$$


The fact that to $\mathscr{P}_{-k} \equiv \mathscr{P}_{k} \times \mathscr{P}_{k}$ is added a space of dimension two of particular vector polynomials of degree $(k+1)$ has to do with the following : if $T_{h}$ (resp. $S_{h}$ ) denotes the finite dimensional subspace approximating $H(d i v ; \Omega$ ) (resp. $\left.L^{2}(\Omega)\right)$, the numerical analysis of the resulting methods depends on the fact that div $I_{h} \subset S_{h}$. Clearly this is satisfied here since div $\mathscr{P}_{-k} \subset \mathscr{P}_{k-1}$ and since the extra two terms have been chosen so that the divergences of the monomials of degree $k+1$ cancel out. In the previous example, the two components of $\vec{v}$ were determined independently : here this is no longer the case as $v_{1}$ and $v_{2}$ are dependent on each other, a situation we have attempted to illustrate in Fig. 7 .

Baslcally, the two-dimensional vector space added to $k$ adds four extra terms to the Pascal triangles which are coupled two by two as follows : a term in position $(k+1,0)$ (resp. $(1, k)$ ) for $v_{1}$ coupled to a term in position $(k, 1)$ (resp. $(0, k+1))$ for $v_{2}$. These two pairs of terms are coupled in the sense that if one of them disappears, the other one must also disappear. The unisolvence proof is now easy : first, we get rid of the two trunks ( $P_{-k-2}$ ), then we look at the $(k+1)$ left legs of the Pascal triangle corresponding to $v_{1}$. The leftmost leg has three bones (!) so we can say nothing about $i t$, but the $k$ following ones have only two bones which must disappear by parity considerations as in the proof of our basic algorithin, implying in particular that the $(1, k)$ position must be vold, ant also the $(0, k+1)$ one for $v_{2}$ thanks to the coupling. Looking now at the $(k+1)$ rlght legs of the Pascal triangle corresponding to $v_{2}$, we see that they are now all with two bones since the third bone of the rightmost leg has been canceled. Consequently they are all void, in particular in the $(k, 1)$ position. Implying that the $(k+1,0)$ position for $v_{1}$ is void and that finally the leftmost leg of $v_{1}$ also disappears QED.

We remark that for the construction of the basis for $s_{k}$ our algorithm applies up to the point where we take into account the dependance of the two components of the vector variable. 


\section{IV - NODAL FINITE ELEMENTS OF THE BDM TYPE}

Example 5: The nodal finite elements of the Brezzi-Douglas-Marini (BDM) type [4].

In Arnold-Brezzi [1], a mixed-hybrid formulation of the f.e.m. was presented which has many computational advantages over the classical mixed one [25]. In fact in the mixed-hybrid formulation, the conformity conditions in $H(d i v ; \Omega)$ are dropped $\left(T_{h} \in H(d i v ; \Omega)\right)$ and enforced by the use of Lagrangien parameters. These parameters are associated with noments of the normal component of $\vec{v}$ on the edges of the cells and therefore provide some valuable information about the corresponding moinents of the dual variable, i.e. the scalar variable u. Hence edge information can be combined with the cell information concerning $u$ in a postprocessing operation performed cell by cell, to obtain a better approximation of $u$ than the original one in $L^{2}$. This procedure can be called an "extension" or "enhancement" of $u$.

In their original paper [4], Brezzi, Douglas and Marini proposed such a nodal extension for their family of mixed finite elements. However, except in the case $k=1$, their extension, does not use all the information available. In fact, the number of parameters used is $\left(k^{2}+5 k+12\right) / 2$ while if all the parameters were used, namely $4(k+1)$ edge moments plus $k(k+1) / 2$ cell moments, the total number of parameters would be $\left(k^{2}+9 k+8\right) / 2$. Except for $k=1$, this number is larger than the previous one. Since this information is available, why not use it completely. The convergence order will not be modified but the approximation of $\Lambda$ will in principle be richer.

Our general algorithm can aga in be applied and we have here (see Fig. 8)

$$
\begin{gathered}
D_{k}=\left\{m_{L}^{1}\left(u_{h}\right), m_{R}^{i}\left(u_{h}\right), m_{D}^{i}\left(u_{h}\right), m_{U}^{i}\left(u_{h}\right), \quad i=0, \ldots, k,\right. \\
\left.m_{C}^{1 j}\left(u_{h}\right), 0 \leqq i+j \leqslant k-1\right\}, \operatorname{card}\left(D_{k}\right)=\left(k^{2}+9 k+8\right) / 2, \\
S_{k}=\mathscr{P}_{k+1} \oplus \mathscr{Q R}_{k} \oplus \operatorname{span}\left\{P_{i i}, P_{i+1, i}, P_{i, i+1}, P_{i+2, i}-P_{i, i+2}:\right. \\
(k+1) / 2<i \leq k\},
\end{gathered}
$$

where

$$
\begin{aligned}
& \mathscr{R}_{k}=\operatorname{span}\left\{P_{i+1, i}, P_{i, i+1}, P_{i+2, i}-P_{i, i+2}, i=k+1 / 2\right\}, k \text { odd, } \\
& \qquad P_{\left.i+2, i^{-P} P_{i+2}: i=k / 2\right\}, k \text { even, }} \\
& \forall k \in \mathbb{N}
\end{aligned}
$$


Note that we do not restrict $k$ to $\mathbb{N}_{*}$ but only to $\mathbb{N}$, as a nodal finite element of the BDM type exists per se when $k=0$ even if it cannot be directly related by extension to a mixed-hybrid. BDM finite element. Also when $k=1$, the spaces $S_{1}$, we construct is identical to the one Brezzi-Douglas-Marini considered for their nodal extensions, another positive feature of our alyorithm. Finally as it is easy to check, these finite elements climb correctly in order.

It is tempting to investigate the possibility of constructing other families of nodal finite elements with the BDM philosophy of minimizing the number of parameters necessary to ensure the same orders of convergence. To pass the patch test at order $k$, we use $(k+1)$ moments on each edge or $4(k+1)$ such moments in total. To ensure $\mathscr{P}_{k+1} \subset S$, we need a total, edge and cell moments, of $(k+2)(k+3) / 2$ degrees of freedom at least. In Table $I$, we show these numbers for the first six values of $k(k=0, \ldots, 5)$.

We remark that for $k \leqq 3$ the number of edge moments necessary to satisfy the patch test is greater than the number of edge and cell moments necessary to satisfy $\mathscr{P}_{k+1} \subset S$, and the situation is reversed when $k \geq 4$. In the same Table $I$, we also give the numbers of degrees of freedom needed by the nodal finite elements of the RTN and BDM type. At this stage, it is important to mention that, in real life calculations, high order elements (say $k>2$ ) are quite seldom used.

It is clearly possible to improve upon the nodal finite elements of the BDM type by taking fewer cell moments, for instance moments with respect to $\mathscr{P}_{k-2}(k \geq 1)$ (family a) or with respect to $\mathscr{P}_{k-3}(k \geq 2)$ (family b) instead of with respect to $\mathscr{P}_{k-1}(k \geqq 0)$. If we apply Algorithm 1 to obtain the corresponding space $S_{k}$, these spaces are $D_{k}$-unisolvent but the corresponding finite elements do not climb correctly in order as $\mathscr{P}_{k+1}$ is not in general contained in $S_{k}$ (see Figs. 9 and 10).

We can however modify Algorithm 1 in order to achieve correct orders, and we have indicated some of the resulting schemes in Figs. 11. and 12. There does not seem however to be a general pattern for doing so; see Fig. 13. Still, as can be seen for these examples, the kind of unisolvence proof used before is quite helpful. 
Before leaving this section, we illustrate in Fig. 14 that our algorithm can also be used in situations where some asymmetry is present, as for instance when more horizontal moments are prescribed than vertical ones. There are indeed physical situations where the use of thick or thin rectangles is clearly recommendable, as for instance in neutron transport deep penetration problems [15]. 


\section{V - THE THREE-DIMENSIONAL CASE}

In three dimensions, the Pascal triangle becomes a Pascal tetrahedron and it is clearly more difficult to visualize what happens. As before, we shall first assume that on the left and right (or vertical) faces of a given cell, moments with respect to $\mathscr{P}_{k_{x}}$ or $\mathscr{Q}_{k_{x}, l}$ are prescribed, where $k_{x}$ is an integer $\geq-1$, while $\ell_{x}$ when it is specified is a nonnegative integer, a value of $(-1)$ for $k_{x}$ indlcating that no moments are considered on the vertical faces. On the up and down (or horizontal) faces as well as on the front and back (or Prontal) faces, moments are similarly prescribed with respect to $\mathscr{P}_{k}$ or $\mathscr{Q}_{k_{y}, l_{y}}$ and $\mathscr{Q}_{k_{z}}$ or $\mathscr{Q}_{k_{z}, l_{z}}$. Finally, cell moments with respect to $\mathscr{Q}_{m}^{y}$ or Q $\mathrm{m,n}, \mathrm{o}$ are also prescribed. Clearly, one at least of $\mathrm{k}_{\mathrm{x}}, \mathrm{k}_{\mathrm{y}}, \mathrm{k}_{\mathrm{z}}$ and $\mathrm{m}$ should be nonnegative. The notation used is illustrated in Fig. 15. For the sake of simplicity we shall consider the case where $k_{x}=k_{y}=k_{z}=k$ and $\ell_{x}=\ell_{y}=\ell_{z}=\ell$.

In $3 D$, our algorithm can be stated as follows :

Assuine that vertical, horizontal, and frontal face moments with respect to $\mathscr{P}_{k}$ or $\mathscr{Q}_{k, l}$ are specified as well as cell moments with respect to $\mathscr{E}_{\text {in }}$ or $Q_{m, n, o^{\prime}}$ one at least of the integers $k$ and $m$ being nonnegative. The following algorithm gives a corresponding unisolvent basis that we shall represent with the help of a Pascal tetrahedron. We have :

\section{ALGORITHM 2}

(1) All the polynomials $P_{i j k}$ belonging to the specified set $\mathscr{P}_{m}$ or $Q_{m, n, 0}$ must be first included in the basis of $S$. In the pascal tetrahedron, these polynomials will correspond to the ijk positions and we shall say that they constitute the trunk of the basis.

(ii) Outside of the area occupied in the tetrahedron by the trunk, $\rho$ two-boned legs must be pulled out in the $x$-direction, in the $y$-direction, and in the $z$-direction where $p$ is the dimension of the specified set $\mathscr{P}_{k}$ or $\mathscr{C}_{k, \hat{x}}$. 
More precisely, the $2 \mathrm{p}$ vertical, horizontal, and frontal face moments, give $r i s e$ to the basis elements $P_{a_{i j}, i j}$ and $P_{a_{i j}+1, i j}, P_{i j} \in \mathbb{P}_{k}$ or $Q_{k, l}$, $P_{i, b}, j$ and $P_{i, b},+1, j, P_{i j} \in \mathscr{Q}_{k}$ or $\mathscr{Q}_{k, l}$, and $P_{i j, c_{i j}}$ and $P_{i j, c_{i j}+1}$, $P_{i j} \in \mathscr{P}_{k}$ or $\mathscr{Q}_{k, l}$, where the $a_{i j}{ }^{\prime} s, b_{i j}{ }^{\prime} s$, and $c_{i j}{ }^{\prime} s$ indicating the point of departure of the $x$-legs, $y$-legs, and $z$-legs respectively are determined as follows :

For $P_{i j} \in \mathscr{P}_{k}$ or $\mathscr{Q}_{k, l}, a_{i j}$ is the least integer $a, a \geqq \max \{i, j\}$, such that $P_{a i j}$ is not included in the trunk. The $b_{i j}$ 's and $c_{i j}$ 's are defined analogously. We remark that as in the $2 D$ case, the $1 j x-l e g$ and the $i^{\prime} j y^{\prime} y-l e g$ can overlap only if $i^{\prime} i^{\prime}, j=j^{\prime}$ and the points of departure of the legs is the same, $1 . e . a_{i j}=b_{i \prime} j^{\prime}=1=1^{\prime}$, and analogously for $x$-legs and $z$-legs or $y$-legs and $z$-legs.

(iil) If it happens that the point of departure of two but not three legs, say an $x-l e g$ and a $y-l e g$, corresponding to the $1 j$ vertical face nonent and t $\sigma$ the $i j$ horizontal face moment respectively, $i \neq j$, is common to both legs, these two legs will now be attached to the $i$ ij position, and we shall include in the basis in addition to $P_{i j j}, P_{i+1, i j}$ and $P_{i, i+1, j}$ the element $P_{i+2, i j} P_{i, i+2, j^{*}}$ We shall say that $P_{i+2, i j}$ and $P_{i, i+2, j}$ are bones of the $i j x-l e g$ and the $i j$ $y$-leg respectively and that $P_{i i j}$ is the $i i j$-pelvis. The other combinations of two joined legs are handled analogously.

(iv) We still have to consider the case that the point of departure of three legs, an $x-l e g$, a $y$-leg, and a $z$-leg corresponding to say the $i \mathrm{i}$ vertical face moments, the if horizontal face moments, and the ii frontal face moments respectively, is the same. These three legs will now be attached to the iif position, and we shall include in the basis in addition to $P_{i i i}, P_{i+1, i j}$, $P_{i, i+1, i}$ and $P_{i i, i+1}$ the elements $P_{i+2, i i}, P_{i, i+2, i}$, and $P_{i i, i+2}$ with the condition $c_{i+2, i 1}+c_{i, i+2, i}+c_{1 i, 1+2}=0$ where $c_{a b c}$ is the coefficient of $P_{\text {abc }}$ in the expansion of a member $u_{h}$ of $S$, namely

$$
u_{h}=\sum_{a b c} C_{a o c} P_{a b c} .
$$


Then we have :

\section{LEMMA 2}

Given a set $D$ of degrees of freedom conforming to the stated hypotheses, the space $S$ constructed by the above algorithm is unisolvent with respect to $D$.

\section{PROOF}

Quite similar to the proof of Lemma 1.

Remark 1 : As for Algorithm 1, we could have assumed in (i) that $P_{i j k}$ belongs to a set of the form $\mathscr{Q}_{m, n, 0} \cup Q_{0, m, n} \cup Q_{n, 0, m}$ in order to be able to construct Langenbuch-Maurer-Werner $[19,20] \quad \Sigma$ or sum nodal schemes in 3D, in analogy with example 1. Again, these schemes do not climb correctly in order and their interest is therefore limited.

Remark 2: In algorithm 2, we could also include asymmetric cases $k_{x} \neq k_{y} \neq k_{z}$ (and possibly $\ell_{x} \neq \ell_{y} \neq \ell_{z}$ ). In such situations an analog to point (iv) of Algorithm 1 should also be considered. In practice, when such a situation is encountered, it is trivial to proceed by analogy with the 2D case. 
VI - SOME EXAMPLES IN 3D

Example 6: The Hennart nodal schemes in 3D [17].

These schemes are obtained from the mixed-hybrid formulation à la Arnold-Brezzl [1] of the Nédélec [22] mixed finite elements (see Example 7), under appropriate nypotheses. See Ref. 6 for the 20 case. We have :

$$
\begin{aligned}
& D_{l}=\left\{m_{v}^{1 j}\left(u_{n}\right), m_{H}^{1 j}\left(u_{n}\right), m_{F}^{1 j}\left(u_{h}\right), \quad 1, j=0, \ldots, l,\right. \\
& m_{C}^{l j k}\left(u_{n}\right), \quad 1, j, k=0, \ldots, l l \text {, } \\
& \operatorname{card}\left(D_{\ell}\right)=(\ell+1)^{2}(\ell+7) \\
& s_{\ell}=\mathscr{Q}_{\ell+2, \ell, \ell} \quad \mathscr{Q}_{\ell, \ell+2, \ell} \quad \mathscr{Q}_{\ell, \ell, \ell+2} \\
& \operatorname{dim}\left(S_{\ell}\right)=(\ell+1)^{2}(\ell+7) \quad \\
& \forall \ell \in \mathbb{N} \text {. }
\end{aligned}
$$

Explicit dual basis functions are given in [17]. These schemes are illustrated in Figs. 16 and 17 and clearly climb correctly in order.

Example 7: The original Nédélec mixed finite elements [22].

The case of the scalar varlable is as trivial in $3 D$ as it is in $2 D$ and we shall only consider the veotor varlables $\vec{v}=\left(v_{1}, v_{2}, v_{3}\right)$. For $\vec{v}$

$$
\begin{aligned}
& D_{\ell}=1 m_{H}^{l j}\left(v_{h 1}\right) \quad, \quad 1, j=0, \ldots, \ldots, \\
& \mathrm{m}_{\mathrm{C}}^{1 j k}\left(\dot{v}_{h 1}\right) \quad, \quad 1=0, \ldots, \ell-1 ; j, k=0, \ldots, l, \\
& m_{v}^{1 j}\left(v_{h 2}\right) \quad, \quad 1, j=0, \ldots, l \text {, } \\
& m_{C}^{1 j k}\left(\dot{v}_{n 2}\right) \quad, \quad j=0, \ldots, l-1 ; 1, k=0, \ldots, l, \\
& m_{F}^{1 j}\left(v_{h 3}\right) \quad, \quad i, j=0, \ldots, l \text {, } \\
& \mathrm{m}_{\mathrm{C}}^{1 j \mathrm{k}}\left(\mathrm{v}_{\mathrm{n} 3}\right) \quad, \quad k=0, \ldots, \ell-1 ; i, j=0, \ldots, l l \text {, } \\
& \text { card } D_{\ell}=3(\ell+1)^{2}(\ell+2)
\end{aligned}
$$




$$
\begin{aligned}
& S_{\ell}=\left\{\mathcal{Q}_{\ell+1, \ell, \ell} \times \mathscr{Q}_{\ell, \ell+1, \ell} \times Q_{\ell, \ell, \ell+1}\right\}, \operatorname{dim} S_{\ell}=3(l+1)^{2}(\ell+2), \\
& \quad \forall \quad \ell \in \mathbb{N}
\end{aligned}
$$

See Figs. 18 and 19. As shown by Nédélec [22], these elements climb correctly in order. 
Example 8: The new Nédélec mixed finite elements [23].

For the scalar variable in the same as that in example 7, while for the vector variable $\vec{v}=\left(v_{1}, v_{2}, v_{3}\right)$, we shall have

$$
\begin{aligned}
& D_{\ell}=\left\{m^{i j}\left(v_{1 h}\right) \quad, i, j=0, \ldots, l,\right. \\
& m_{C}^{i j k}\left(v_{1 h}\right) \quad, i=0, \ldots, l-2 ; j, k=0, \ldots, l, \\
& \mathrm{~m}^{i \mathrm{j}}\left(\mathrm{v}_{2 h}\right) \quad, \quad 1, j=0, \ldots, l \text {, } \\
& m_{C}^{i j k}\left(v_{2 h}\right) \quad, j=0, \ldots, l-2 ; i, k=0, \ldots, l, \\
& m^{i j}\left(v_{3 h}\right) \quad, i, j=0, \ldots, l \text {, } \\
& \left.m_{C}^{i j k}\left(v_{3 h}\right) \quad, k=0, \ldots, l-2 ; i, j=0, \ldots, l\right\} \text {, } \\
& \text { card } D_{\ell}=3(l+1)^{3} \\
& s_{\ell}=\mathscr{Q}_{\ell} \times \mathscr{Q}_{\ell} \times \mathscr{Q}_{\ell}, \operatorname{dim} s_{\ell}=3(\ell+1)^{3}, \\
& \forall \ell \in \mathbb{N}_{*} \cdot
\end{aligned}
$$

See Fig. 20. As shown by Nédélec [23], these elements clininb correctly in order. If we call $N_{1}$ the dimension of $S_{\ell}$ (or the cardinality of $D_{\ell}$ ) for the original Nédélec mixed inite elements and $\mathrm{N}_{2}$ the corresponding dimension for the new Nédélec mixed finite elènents, we have

$$
\frac{N_{2}}{N_{1}}=\frac{\ell+1}{\ell+2},
$$

which tends asymptotically to one. Consequently, the lowest order new Nédélec mixed finite elements with $l=1$ or maybe $l=2$ are the most interesting ones. We note that if a mixed-hybrid form is considered, the nodal finite elements obtained by extension would be identical to the Hennart nodal schemes in 3D [17] as are those obtained from example 7 . 
Example 9 : The 3D mixed finite elements "à la BDM" [3].

In a recent. paper [3], Brezzi et al. proposed mixed finite elements in $3 D$, which are a natural generalization to $3 D$ of the $2 D$ mixed finite elements of Ref. [4] (see Example 4).

Assuming that $\ell \in \mathbb{N}_{*}$, the scalar variable is taken to be in $\mathscr{Q}_{\ell-1}$, instead of $Q_{\ell}$, namely

$$
\begin{gathered}
D_{\ell}=\left\{m_{c}^{i j k}, 0 \leqq i+j+k \leqq \ell-1\right\} \text {, card } D_{\ell}=\frac{\ell(\ell+1)(\ell+2)}{6}, \\
S_{\ell}=\mathscr{P}_{\ell-1}, \quad d i m S_{\ell}=\frac{\ell(\ell+1)(\ell+2)}{6} \\
\forall \ell \in \mathbb{N}_{*}
\end{gathered}
$$

For the vector variable $\vec{v}=\left(v_{1}, v_{2}, v_{3}\right)$

$$
\begin{aligned}
& D_{\ell}=\left\{m_{H}^{i j}\left(v_{h 1}\right) \quad, \quad 0 \leqq i+j \leqq \ell\right. \text {, } \\
& m_{C}^{i j k}\left(v_{h 1}\right) \quad, \quad 0 \leqq i+j+k \leqq \ell-2 \text {, } \\
& m_{v}^{i j}\left(v_{h 2}\right) \quad, \quad 0 \leqq i+j \leqq \ell, \\
& m_{C}^{i j k}\left(v_{h 2}\right) \quad, \quad 0 \leqq i+j+k \leqq \ell-2 \text {, } \\
& m_{F}^{i j}\left(v_{h s}\right) \quad, \quad 0 \leqq i+j \leqq \ell, \\
& \left.m_{C}^{i j k}\left(v_{h 3}\right) \quad, \quad 0 \leqq i+j+k \leqq \ell-2\right\} \text {, } \\
& \operatorname{card} D_{\ell}=\left(\ell^{3}+6 \ell^{2}+17 \ell+12\right) / 2 \text {, } \\
& s_{\ell}=\left\{\mathscr{P}_{\ell} \times \mathscr{P}_{\ell} \times \mathscr{P}_{\ell}\right\} \oplus \operatorname{span}\left\{\left(\begin{array}{c}
P_{\ell+1,00} \\
-(\ell+i) P_{\ell 10} \\
0
\end{array}\right),\left(\begin{array}{c}
-(2 \ell+1) P_{10 \ell} \\
0 \\
P_{00,2 \ell+1}
\end{array}\right),\left(\begin{array}{c}
P_{0, \ell+1,0} \\
-(\ell+1) P_{0 \ell 1}
\end{array}\right)\right\} \\
& \underset{i=1, \ldots, l}{\oplus \operatorname{span}}\left\{\left(\begin{array}{c}
i+1) P_{1 i, \ell-i} \\
-P_{0, i+1, \ell-i}^{\prime} \\
0
\end{array}\right) \cdot\left(\begin{array}{c}
0 \\
-P_{i+1, \ell-i, 0} \\
0 \\
(i+1) P_{i, \ell-i, 1}
\end{array}\right) \cdot\left(\begin{array}{c}
(i+1) P_{\ell-i, 1 i} \\
-P_{\ell-1,0, j+1}
\end{array}\right)\right\} \\
& , i=1, \ldots, l,
\end{aligned}
$$




$$
\operatorname{dim} s_{\ell}=\left(\ell^{3}+6 \ell^{2}+17 \ell+12\right) / 2
$$

As in Example 4, it is possible to use our general technique to prove unisolvence,

Example 10 : The (new) nodal finite elements of BDM type in 3D.

These nodal finite elements are extensions "à la Arnold-Brezzi" of the 3D mixed finite elements "à la BDM" of Example 9. We have :

$$
\begin{aligned}
& D_{\ell}=\left\{m_{H}^{i j}\left(u_{h}\right), \quad m_{V}^{i j}\left(u_{h}\right), m_{F}^{i j}\left(u_{h}\right), \quad 0 \leqq i+j \leq \ell,\right. \\
& \left.m_{C}^{i j k}\left(u_{h}\right), \quad 0 \leqq i+j+k \leq \ell-1\right\} \text {, } \\
& \text { card } D_{\ell}=(l+1)(l+2)(l+18) / 6 \\
& S_{\ell}=\mathscr{Q}_{\ell-1} \oplus \underset{i=1}{\oplus} A_{i} \oplus \underset{i=1}{\oplus} B_{i} \oplus c,
\end{aligned}
$$

where $A_{1}, A_{2}$ and $A_{3}$ are the spaces generated by the solitary legs corresponding to the vertical, horizontal, and frontal face moments respectively,

$$
A_{1}=\operatorname{span}\left\{P_{i j k}, P_{i+1, j, k}: i>j, k, 1+j+k=l\right\}
$$

with $A_{2}$ and $A_{3}$ being defined as was $A_{1}$ with the obvious rotation of the indices: $B_{1}, B_{2}$ and $B_{3}$ are spaces generated by the jolned pairs of legs,

$$
\begin{aligned}
& B_{1}=\operatorname{span}\left\{P_{i j j}, P_{i, j+1, j}, P_{i j, j+1}, P_{i, j+2, j}-P_{i j, j+2}:\right. \\
& i>j, l \leq 2 i+j \leq 2 \ell-j\},
\end{aligned}
$$

again with $B_{2}$ and $B_{3}$ being defined as was $B_{1}$ with the proper index rotation: and $C$ is the space generated by the joined triples of legs, 


$$
\begin{aligned}
& C=\operatorname{span}\left\{P_{i 1 i}, P_{i+1, i, i}, P_{i, i+1, i}, P_{i, i, i+i}, P_{1+2,1, i},\right. \\
& \quad P_{i, i+2, i}, P_{i, i, i+2}, \text { with } C_{i+2, i i} \\
& \left.\quad C_{1, i+2, i}+C_{i i, i+2}=0: \ell / 3 \leqq i \leqq \ell / 2\right\} . \\
& \operatorname{dim} S_{\ell}=(\ell+1)(\ell+2)(\ell+18) / 6 . \\
& \forall \ell \in \mathbb{N}
\end{aligned}
$$

The first two such elements are illustrated in Figs. 21 and 22, Including the case $\ell=0$ which exists per se. To see that these elements again climb correctly in order, we need to show that $\mathscr{Q}_{\ell+1} \subset S_{\ell}$. Consider $P_{i j k}$ with $i+j+k=\ell$. We may assume $1 \geq j, k$. Then $P_{i j k}$ is the first bone of the leg corresponding to the $j k$ horizontal moments : if $i>j, k ; P_{i j k} \in A_{1}$, if $i=j>k$ or $I=k>j$, $P_{1 j k} \in B_{3}$ or $P_{1 j k} \in B_{2}$, and if $i=j=k, P_{i j k} \in C$. Next suppose $1+j+k=l+1$ and again assume $1 \geq j, k$. If $i>j, k$ then $P_{i j k}$ is the second bone of the leg corresponding to the $j k$ vertical moments. Otherwise, say $i=j, P_{i j k}$ is the first bone for the ik horizontal face moments and for the $j k$ vertical face moments and $P_{i j k} \in B_{3}$ unless, of course, $i=j=k$ in which case $P_{i j k} \in C$.

We remark that since $\mathscr{P}_{\ell+1} \subset S_{\ell}$ an expression analagous to that given for the 2D BDM nodal elements could be used to define $S_{\ell}$ but in $3 D$ such an expression is quite tedious. (In fact even with the expression given here $s_{l}$ is probably more easily constructed using Fig. 21 or Fig. 22 than using the formula.) 


\section{A P PENDIX A}

\section{Examples of basis functions in 20}

\section{1 - The Hennart nodal schemes in 2D $[17,18]$.}

The basis functions have been given explicltly earlier [17] and will be recalled here for the sake of completeness.

copresponding to the degrees of freedom $\mathrm{m}^{i}$ and $\mathrm{m}_{\mathrm{C}}^{\mathrm{ij}}$ of $\mathrm{u}$ (see Eq. (13)), we have :

$$
\begin{array}{ll}
u_{L}^{i}=\frac{1}{2}(-1)^{k+1}\left[P_{k+1, i}-P_{k+2, i}\right], & i=0, \ldots, k, \\
u_{R}^{i}=\frac{1}{2}\left[P_{k+1, i}+P_{k+2, i}\right], & i=0, \ldots, k, \\
u_{C}^{i j}=P_{i j}-P_{k+m(i), j}-P_{i, k+m(j)}, i, j=0, \ldots, k,
\end{array}
$$

where $m(1)$ (resp. $m(j))=1$ or 2 is such that $i$ and $k+m(i)$ (resp. $j$ and $j+m(j)$ ) have the same parity. As in the preceeding examples, the basis functions corresponding to the vertical degrees of freedom $m^{i}$ can be obtained by excinanging $x$ and $y$ and by replacing $L$ and $R$ by $D$ and $U$ respectively. In [17], asymmetric $2 D$ basis functions are also given.

\section{2 - The Raviart-Thomas mixed finite elements [25].}

These basis functions have also been given explicity earlier [16].

For the degrees of freedom $m_{C}^{i j}$ of the scalar variable $u$ (see Eq. (16)). the basis functions are quite simple (they are of the trunk-only type) and reduce to

$$
u_{C}^{i j}(x, y) \equiv u_{C}^{i j}=P_{i j} \equiv P_{i j}(x, y) \ldots, \ldots 1, j=0, \ldots, k .
$$

For the degrees of freedom $\mathrm{m}^{i}$ and $\mathrm{m}_{\mathrm{C}}^{i j}$, of the first component $\mathrm{v}_{1}$ of the vector variable $\vec{v}$ (see Eq. (17)), we have the corresponding basis functions 
and

$$
\begin{aligned}
& v_{1 L}^{i}=\frac{1}{2}(-1)^{k}\left[P_{k+1,1}-P_{k i}\right], \\
& v_{1 R}^{1}=\frac{1}{2}\left[P_{k i}+P_{k+1, i}\right], \quad, \quad i=0, \ldots, k,
\end{aligned}
$$

$$
v_{1 C}^{i j}=P_{i j}-P_{k-1+m(1), j} \quad, \quad i=0, \ldots, k-1, j=0, \ldots, k \text {, }
$$

where $m(i)=1$ or 2 is such that 1 and $k-1+m(i)$ have the same parity. Simflar expressions hold for the corresponding degrees of freedom of $v_{2}$, with the roles of $x$ and $y$ excinanged and $L$ and $R$ replaced by $D$ and $U$ respectively.

\section{3 - The Brezzi-Douglas-Marini mixed Pinite elements [4].}

Here the situation is more complex than in the Raviart-Thomas case and for the vector varlable, we were unable to find, a general compact expression. For the scalar variable formula (A2) is again valid.

We can however easily retrieve the basis functions by hand for the simplest cases or by computer as soon as $k$ grows. For $k=1$ for instance (the first such inixed inite element), we have following (19)

$$
\begin{aligned}
& \vec{v}_{L}^{0}=\left(\frac{1}{2}\left(P_{10}-P_{00}\right), 0\right) \\
& \vec{v}_{R}^{0}=\left(\frac{1}{2}\left(P_{00}+P_{10}\right), 0\right) \\
& \vec{v}_{L}^{1}=\left(\frac{1}{2}\left(P_{11}-P_{01}\right), \frac{1}{6}\left(P_{00}-P_{02}\right)\right) \\
& \vec{v}_{R}^{1}=\left(\frac{1}{2}\left(P_{01}+P_{12}\right), \frac{1}{6}\left(P_{00}-P_{02}\right)\right) .
\end{aligned}
$$

Similar expressions for $\vec{v}_{D}^{0}, \vec{v}_{U}^{0}, \vec{v}_{D}^{1}$ and $\vec{v}_{U}^{1}$ are obtained by exchanging the components as well as $x$ and $y$ and by replacing $L$ and $R$ by $D$ and $U$ respectively.

4 - The nodal finite elements of the BDM type in 20.

Here agaln, a general pattern was not evident but we have for instance corresponding to the degrees of freedom given by (20) and in the case $k=0$ 


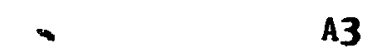

$$
\begin{aligned}
& u_{L}^{0}=\frac{1}{4}\left(P_{00}-2 P_{10}+P_{20}-P_{02}\right) \\
& u_{R}^{0}=\frac{1}{4}\left(P_{00}+2 P_{10}+P_{20}-P_{02}\right),
\end{aligned}
$$

with $u_{D}^{0}$ and $u_{U}^{0}$ being determined simllarly.

In the case $k=1$, we get

$$
\begin{aligned}
& u_{L}^{0}=\frac{1}{2}\left(-P_{10}+P_{20}+P_{12}\right) \\
& u_{R}^{0}=\frac{1}{2}\left(+P_{10}+P_{20}-P_{12}\right) \\
& u_{L}^{1}=\frac{1}{4}\left(-P_{11}+2 P_{21}-P_{31}+P_{13}\right) \\
& u_{R}^{1}=\frac{1}{4}\left(+P_{11}+2 P_{21}+P_{31}-P_{13}\right) .
\end{aligned}
$$

and

$$
u_{C}^{00}=P_{00}-P_{20}-P_{02}
$$

the same remark as above being valid for the vertical edge basis functions. 


\section{APPENDIX B}

\section{Examples of basis functions in 3D.}

\section{1 - The Hennart nodal schemes in 3D [17,18].}

These basis punctions were given in [17] and are recalled here, Corresponding to the degrees of freedom $m_{H}^{1 j}$ and $m_{C}^{1 j k}$ of $u$ as in (22), we have

$$
\begin{array}{ll}
u_{L}^{1 j}=\frac{1}{2}(-1)^{\ell+1}\left[P_{\ell+1,1 j}-P_{\ell+2,1 j}\right] & 1, j=0, \ldots \ldots l, \\
u_{R}^{1 j}=\frac{1}{2}\left[P_{\ell+1,1 j}+P_{\ell+2,1 j}\right] & 1, j=0, \ldots \ldots l, \\
u_{C}^{1 j k}=P_{1 j k}-P_{\ell+m(1), j k} P_{1, \ell+m(j), k}-P_{i j, \ell+m(k)}, & 1, j, k=0, \ldots, \ell,
\end{array}
$$

where $m(1), m(j)$ and $m(k)$ have the same meaning as before. Again the other face basis functions can be retrleved from $u_{L}^{1 j}$ and $u_{R}^{1 j}$, by trivial symmetry operations.

\section{2 - The Medglec mixed rinite elenents [.22].}

The case of the scalar variable being again trivial, we shall only pay attention to the vector variable and more. specifically to its first component $v_{1}$. The basis functions for the other two components are obtained as in Appendix A by trivial exchanges of the arguments and substitutions of the race Identiflers.

For $v_{1}$ whose degrees of freedom $m^{1 j}$ and $m_{C}^{1 j k}$ are described in (23), we have the corresponding basis functions :

$$
\begin{array}{ll}
v_{1 L}^{1 j}=\frac{1}{2}(-1)^{\ell}\left[P_{\ell+1, i j}-P_{\ell i j}\right], & i, j=0, \ldots, \ell, \\
v_{1 R}^{1 j}=\frac{1}{2}\left[P_{\ell+j}+P_{\ell+1, i j}\right] & , \quad i, j=0, \ldots, \ell,
\end{array}
$$

and

$$
v_{1 C}^{1 j k}=P_{1 j k}-P_{\ell-1+m(1), j k} \quad, \quad t=0, \ldots, \ell-1 ; i, k=0, \ldots, p,(R ?)
$$

where $m(i)=1$ or 2 is such that 1 and $\ell-1+m(i)$ have the same parity. 


\section{The new Hédélec mlxed finite elements [23].}

As in the previous example, we shall only consider the first component $v$, of the vector varlable whose degrees of freedom $m^{1 j}$ and $m_{C}^{1 j k}$ are described in (24).

The corresponding basis 'functions are

$$
\begin{aligned}
& v_{1 L}^{1 j}=\frac{1}{2}(-1)^{\ell-1}\left[P_{\ell, i j}-P_{\ell-1, i j}\right], \quad 1, j=0, \ldots, l, \\
& v_{1 R}^{1 j}=\frac{1}{2}\left[P_{\ell-1, i j}+P_{\ell i j}\right] \quad, \quad 1, j=0, \ldots, \ell,
\end{aligned}
$$

and

$$
\begin{aligned}
& v_{1 C}^{i j k}=P_{1 j k}-P_{\ell-2+m(1), j k} \quad, \quad 1=0, \ldots, \ell-2 \text {; } \\
& j, k=0, \ldots, l
\end{aligned}
$$

where $m(i)=1$ or 2 is such that $i$ and $\ell-2+m(1)$ have the same parity.

4. The nodal fintte elements of the BDM type in 3D.

As in Appendix A - Section 4, a general pattern was not evident but we have for Instance corresponding to the degrees of freedom given by (26) and In the case $\ell=0$

$$
\begin{aligned}
& u_{L}^{00}=\frac{1}{6}\left(P_{000}-3 P_{100}+2 P_{200}-P_{020}-P_{002}\right), \\
& u_{R}^{00}=\frac{1}{6}\left(P_{000}+3 P_{100}+2 P_{200}-P_{020}-P_{002}\right),
\end{aligned}
$$

The other basts funotions are obtained as above by symmetry considerations. 


\section{AP P ENDIX C}

\section{Error estimates}

In this last appendix, we give a general theorem leading to "error estimates when nodal finite elements are applied to approximate the solution of the second order elliptic equation,

$$
\begin{aligned}
-\Delta u & =r & & \text { in } \Omega, \\
u & =0 & & \text { on } r .
\end{aligned}
$$

Classfcally this strong form of the equation is first transpormed into an equivalent weak form which reads

$$
\begin{aligned}
& \text { Find } u \in v \text { such that } \\
& \qquad a(u, v)=f(v) \quad \forall v \in v,
\end{aligned}
$$

where $V \equiv H_{0}^{2}(\Omega)$. Is the standard Sobolev space of functions which have square integrable (generalized) derivatives up to and including first order and a zero trace on $\Gamma$, while

and

$$
a(u, v)=\int_{\Omega} \vec{\nabla} u \cdot \vec{\nabla} v d \vec{r} \text {, }
$$

$$
f(v)=\int_{\Omega} f v d \vec{r}
$$

To obtain a discretized version of (C2), we assume, as in the text, that we have a fanily indexed by $h$ of regular meshes $\Omega_{h}=\left\{\Omega_{e}: e=1, \ldots E_{h}\right\}$ of the union-of-rectangles type on $\&$ with $h$ an upper bound on the dlameters of the rectangles $\Omega_{e}$ in $\Omega_{h}$. With classical finite element methods, to obtain an algebrale system of finite order $N$, the primal form (C2) is replaced by

$$
\begin{aligned}
& \text { Find } u_{h} \in v_{h} \quad \text { such that } \\
& \quad a\left(u_{h}, v_{h}\right)=f\left(v_{h}\right) \quad \forall v_{h} \in v_{h}
\end{aligned}
$$

where $V_{h}$ is a finite dimensional subspace of $V$ whose elements are polynomials over each $\Omega_{e}$ in $\Omega_{h}$ and are continuous on $\Omega$. 
If nodal finite elements are used instead as in examples $1,2,5,6$ and

10 the elements of the finite dimensional space $V_{h}$ are polynomials over each $\Omega_{e}$ but only some of the moments and not the functions themselves are required to be continuous through the edges (in 2D) or faces (in 3D) common to two adjacent rectangles. On the boundary $r$ moreover, the trace of an element $v_{h} \in v_{h}$ is not necessarily zero but again only some of its moments. Consequently $v_{h} \notin v$ and (C3) must be replaced by

$$
\begin{array}{ll}
\text { Find } & u_{h} v_{h} \text { such that } \\
& a_{h}\left(u_{h}, v_{h}\right)=f\left(v_{h}\right) \quad \forall v_{h} \in v_{h},
\end{array}
$$

where

$$
a_{h}\left(u_{h}, v_{h}\right)=\sum_{l=1}^{E} \int_{\Omega_{e}} \vec{\nabla} u_{h} \cdot \vec{\nabla} v_{h} d \vec{r}
$$

Let us assume in the following that we are in $3 \mathrm{D}$, the $2 \mathrm{D}$ case being trivially deduced from the 3D case, and that the restriction $\left.v_{h}\right|_{\Omega}$ of an element $v_{h}$ of $v_{h}$ to the element $\Omega_{e}$ is defined as in the main text by a set of degrees of freedom $D$ and a space $S$ of polynomials. Assume moreover that $\mathscr{P}_{k} \subset S, k \in \mathbb{N}_{*} \cdot$ and that the restriction $u_{h} l_{\Gamma_{e f}}$ of $u_{h}$ to the faces $\Gamma_{e f}$ of $\Omega_{e}$, where $f$ stands for $L, R, U, D, F$, and $B$ have moments with respect to $\mathscr{E}_{\ell}, \ell \in \mathbb{N}$, continuous through the corresponding faces, i.e. that $m_{f}^{1 j} \in D, 0 \leqq i+j \leq l$. With these hypotheses and assuming that at each step of the following demonstration u has the regularity required by the context, we can prove :

\section{THEOREM}

$$
\left\|u-u_{n}\right\|_{0} \leqq c n^{\lambda+1}\|u\|_{\lambda+1, \Omega}
$$

where $\lambda=\min \{k, \ell+1\}$ and $\|\cdot\|_{0}$ is the $L^{2}$ norm of the error $u-u_{h}$.

In the demonstration which follows, many steps are classical and we shall refer the interested reader to ciarlet [7] for the dat?:?.s. 


\section{Démonstration}

a. Let us first introduce for each element $v_{h} \in v_{h}$ a norm $\left\|v_{h}\right\|_{h}$ defined by

$$
\left\|v_{h}\right\|_{h}=\left.1 \sum_{e=1}^{E}\left|v_{h}\right|_{1, \Omega}^{2}\right|^{\frac{y}{2}}
$$

where

$$
\left|v_{h}\right|_{1, \Omega_{e}}^{2}=\int_{\Omega_{e}} \vec{\nabla} v_{h} \cdot \vec{\nabla} v_{h} d \vec{r}
$$

$\|\cdot\|_{h}$ is a priori only a semi-norm on $v_{h}$. However, we have :

LEMMA 1 : $\|\cdot\|_{h}$ is a norm on $\dot{v}_{h}$.

Proof : $\because\left\|v_{h}\right\|_{h} \Rightarrow 0$ implies that $v_{h / \Omega}=c_{e^{\prime}} e=1, \ldots, E$, where $c_{e}$ is a constant depending in principle on e. Now with the above hypotheses; $c_{e}=v_{h \mid \Omega_{e}}=v_{h / \Omega_{e f}}=m_{f}^{0}\left(v_{h}\right)$ for any face $f$ and is thus continous through it. In other words, $c_{e}=c, \forall e . " F i n a l l y$ on $\Gamma$, at least the zeroth order moment of $v_{h}$ is equal to zero so that $C=0$ QED.

b. Let us enlarge the domain of definition of $a_{h}(\cdot, \cdot)$ and $\|\cdot\|_{h}$ to $v_{h}+v$ :

$* v \in v, a_{h}(v, v)=a(v, v)$ and $\|v\|_{h}=|v|_{1, \Omega}$. We remark that \|\|$_{h}$ is a norm on $v_{h}+V$ and we have

\section{LEMMA 2 :}

For the family of spaces $v_{h}, a_{h}(\cdot, \cdot)$ is uniformly $v_{h}$ elliptic in the sense that there exists $\alpha>0$ such that

$$
\alpha\left\|v_{h}\right\|_{h}^{2} \leqq a_{h}\left(v_{h}, v_{h}\right) \quad \forall v_{h}
$$

where $\alpha$ is independent of $h$. Moreover there exists a positive constant is Independent of $h$ such that

$$
\left|a_{h}(u, v)\right| \leq M\|u\|_{h}\|v\|_{h} \quad \forall u, v \in v_{h}+v \text {. }
$$

Proof: The proof is based on the definitions (C5) and (C7), and is trivial.

As a consequence of this lemma, there is one and only one solution to the

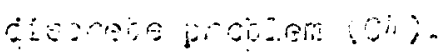


c. The next step consists in getting error estimates and it depends on the second Strang lemma (Ciarlet [7], p. 210), saying that if we consider a family of discrete problems (C4) such that Lemma 2 is satisfied, there exists a constant $C$ independent of $h$ such that

$$
\left\|u-u_{h}\right\|_{h} \leq c\left(\inf _{v_{h} \in v_{h}}\left\|u-v_{h}\right\|_{h}+\sup _{w_{h} \in v_{h}} \frac{\left|a_{h}\left(u, w_{h}\right)-f\left(w_{h}\right)\right|}{\left\|w_{h}\right\|_{h}}\right)
$$

To obtain error estimates, it remains to bound the two terms appearing in (C8), the second one coming from the nonformity $v_{h} \notin v$. We have

(i) For the first term, we clearly have

$$
\inf _{h} \in v_{h}\left\|u-v_{h}\right\|_{h} \leq\left\|u-\hat{u}_{h}\right\|_{h} \text {, }
$$

where $\hat{u}_{h}$ is the interpolation of $u$ in $v_{h}$, distinct in princlple from its approximation $u_{h}$. If $u$ is any member of $\mathscr{E}_{k} \subset s$, we shall have $u \equiv \hat{u}_{h}$ and it is a direct consequence of interpolation theory in Sobolev spaces (Ciarlet [7], 83.1) that under standard assumptions on the regularity of the mesh considered, there exists a constant $C$ such that

so that

$$
\left\|u-\hat{u}_{h}\right\|_{h} \leq c h^{k}|u|_{k+1, \Omega}
$$

$$
\inf _{v_{h} \in v_{h}}\left\|u-v_{h}\right\|_{h} \leqq c h^{k}|u|_{k+1, \Omega}
$$

(1i) For the second term, we have for any $w_{h} \in v_{h}$

and

$$
f\left(w_{h}\right)=\int_{\Omega} f w_{h} d \vec{r}=\sum_{e=1}^{E} \int_{\Omega} f w_{h} d \vec{r},
$$

$$
\int_{\Omega_{e}} f w_{h} d \vec{r}=-\int_{\Omega_{e}} \Delta u \cdot w_{h} d \vec{r}=\int_{\Omega_{e}} \vec{\nabla} u \cdot \vec{\nabla}_{w_{h}} d \vec{r}-\int_{\Gamma_{e}}\left(\vec{\nabla} u \cdot \overrightarrow{1}_{e}\right) w_{h} d s,
$$
where $r_{e}$ is the boundary of $\Omega_{e}$ and $\vec{r}_{e}$ a unit outward pointing normal on $r_{e}$.
Consequently

$$
a_{h}\left(u, w_{h}\right)-f\left(w_{h}\right)=\sum_{e=1}^{E} \int_{\Gamma_{e}}\left(\nabla_{u} \cdot t_{e}^{t}\right) w_{h} d s .
$$


Let $\pi_{\text {ef }}^{\ell}\left(\vec{\nabla} u \cdot \overrightarrow{1}_{e}\right)$ denote the interpolation of ${\vec{\nabla} u \cdot \vec{i}_{e}}_{\mid} \Gamma_{e f}$ in $\mathscr{P}_{\ell}, \& \in \mathbb{N}$. Clearly, the sum appearing in the righthand side of $(C 10)$ is identically zero when $\nabla_{u} \cdot \vec{f}$ is replaced over each face appearing in $\Gamma_{e}$ by this interpolation. First of all,

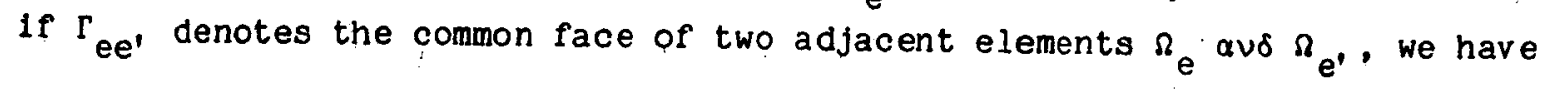

$$
\int_{\Gamma_{e e^{\prime}}} \pi_{e e^{\prime}}^{\ell}\left(\vec{\nabla}_{u_{\Omega_{e}}} \cdot \vec{t}_{e}-\vec{\nabla}_{u_{\Omega_{e}}} \cdot \vec{i}_{e^{\prime}}\right) w_{h} d s=0,
$$

since the moments $m_{f}^{1 j}\left(w_{h}\right), 0 \leq 1+j \leq l$, are continuous through any internal face. Moreover if $\Gamma_{\text {ef }}$ is any external face $\left(\Gamma_{\text {ef }} \subset \Gamma\right.$ ), we have

$$
\int_{e f} \pi_{e f}^{\ell}\left(\vec{\nabla}_{u} \cdot \vec{l}_{e}\right) w_{h} d s=0
$$

as the moments $m_{f}^{i j}\left(w_{h}\right), 0 \leq 1+j \leq \ell$, are zero on any external face. Consequently (C10) can be rewritten as

$$
a_{h}\left(u, w_{h}\right)-f\left(w_{h}\right)=\sum_{e} \int_{e}\left\{\vec{\nabla}_{u} \cdot \vec{t}_{e} e^{\left.-\pi_{e}^{\ell}\left(\vec{v}_{u} \cdot \vec{t}_{e}\right)\right\} w_{h} d s} .\right.
$$

Consider one term of the sum appearing in (C13). It is a linear functional

$$
w_{h} \longrightarrow \int_{\Gamma_{e f}}\left\{\vec{v}_{u} \cdot \vec{\imath}_{e}-\pi_{e f}^{\ell}\left(\vec{\nabla} u \cdot \vec{\imath}_{e}\right)\right\} w_{h} \text { ds }
$$

continuous on $H^{1}\left(\Omega_{e}\right)$, of norm less than or equal to $\left\|\vec{\nabla}_{u} \cdot \vec{i}_{e}-\pi_{e f}^{l}\left(\nabla_{u} \cdot \overrightarrow{1}_{e}\right)\right\|_{0, \Gamma_{e f}}$, Identical to zero $\forall w_{h} \in \cdot \mathscr{P}_{0}$ (since $\vec{\nabla}_{u} \cdot \overrightarrow{1}_{e}$ and its interpolate have at least the same mean value over each $\Gamma_{\text {ef }}$ ). Employing the Bramble-Hilbert lemma, (Bramble-Hilbert [2] Th.2), we have

$$
\left|\int_{\Gamma_{e f}}\left\{\vec{\nabla}_{u} \cdot \vec{i}_{e}-\pi_{e f}^{\ell}\left(\vec{\nabla} u \cdot \vec{f}_{e}\right)\right\} w_{h} d s\right| \leq c h\left|w_{h}\right|_{1, \Omega_{e}}\left\|\vec{\nabla}_{u} \cdot \vec{i}_{e}-\pi_{e f}^{\ell}\left(\vec{\nabla}_{u} \cdot \vec{i}_{e}\right)\right\|_{0, \Gamma_{e f}}
$$

Using again the results of interpolation theory in Sobolev spaces we have

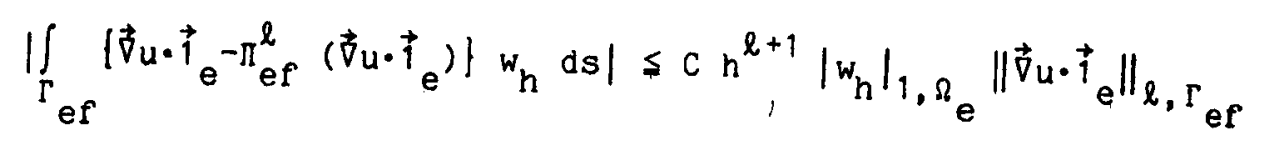

nence

$$
\left|\int_{\Gamma}\left\{\vec{\nabla} u \cdot \vec{l}_{e}-\pi_{e f}^{\ell}\left(\vec{\nabla}_{u} \cdot \vec{t}_{e}\right)\right\} w_{h} d s\right| \leq c n^{\ell+1}\left|w_{h}\right|_{1, \Omega_{e}}\|u\|_{\ell+2, \Omega} \cdot
$$


Combining $\mathrm{C} 14$ and $\mathrm{C} 13$ we obtain

$$
\left|a_{h}\left(u, w_{h}\right)-f\left(w_{h}\right)\right| \leq c n^{\ell+1}\left\|w_{h}\right\|_{h}|u|_{\ell+2, \Omega}
$$

so that

$$
\sup _{w_{h} \in v_{h}} \frac{\left|a_{h}\left(u, w_{h}\right)-f\left(w_{h}\right)\right|}{\left\|w_{h}\right\|_{h}} \leq c h^{\ell+1}|u|_{\ell+2, \Omega} .
$$

Finally, from $(C 8),(C 9)$ and $(C 15)$, we get

$$
\left\|u-u_{h}\right\|_{h} \leq c n^{\lambda}|u|_{\lambda+1, \Omega}
$$

where $\lambda=\min \{k, \ell+1\}$.

d. To get the $L^{2}$ estimate of (C6), standard Aubin-Nitsche arguments should be applied. See for Instance Nitsche [24] and Lesaint [21], QED. 


\section{REFER ENCES}

D.N. ARNOLD and F. BREZZI, Mixed and nonconforming finite element methods: Implementation, postprocessing and error est Imates, $\mathbf{M}^{2} \mathbf{A N}, 19,7-32$ (1985).

J.H. BRAMBLE and S.R. HILBERT, Estimation of linear functionals on Sobolev spaces with application to Fourier transforms and spline interpolation, SIAM J. Numer. Anal. I, 113-124 (1970).

3 F. BREzZI, J. DOUGLAS, Jr., R. DURAN, and M. FORTIN, MIxed finite elements for second order efliptic problems in three variables, Preprint.

4 F. BREZzI, J. DOUGLAS, Jr., and L.D. MARINI, Two familles of mixed $f$ inite elements for second order elliptic problems, Numer. Math., 47, 217-235 (1985).

5. G. Chavent, G. COHEN, and J. JAFFRE, Discontinuous upwinding and mixed finite elements for two-phase flow in reservoir simulation, Comput. Methods App11. Mech. Engrg., 47, 93-118 (1984).

6 G. "CHAVENT and J. JAFFre, Mathematical Models and Finite Blements in Reservolr Simulation, North Holland, Amsterdam (1986).

7 P.G. CIARLET, The Finite Element Hethod for Elliptic Problens. North-Holland, Amsterdam (1978).

8 E. DEL VALLE, J.P. HEMNART and D. MEADE, Finite element formulations of nodal schemes for neutron diffusion and transport problems, Mucl. Scl. Engng., 92, 204-211 (1986).

9 J. DoRurwG, Modern coarse-mesh methods - A development of the '70's, Computational Methods in Nuclear Engineering, Vol. 1, pp, 3.1-3.31. American Nuclear Society, WLillamsburg, Virginia (1979).

10. J. Dovglas, Jr., Numerical methods for the flow of miscible fluids in porous media, Numerical Methods in Coupled Systems, pp. 405-439, R.W. Lewis, P. Bettess, E. Hinton, Eds., John Wiley, New York (1984).

1 Ch. FEDON-MAGNAUd, J.P. HENNART, and J.J. LAUTARD, On the relationshlp between same nodal schemes and the finite element method in static diffusion calculations, Advances in Reactor Computations, Vol. 2 , pp. 987-1000, American Nuclear Society, Salt Lake City, Utah (1983).

12 H. FIMRDAAH, F. BENGWTTZ, and M.F. HAGNER, Interface current techniques for multidimens lonal reactor calculations, Atomkernenergle, 30. 123-128 (1977).

13 R. FRÖHEICH, Summary discussion and sitace or the art review. for coarse-mesh computational methods, Atomkernenergie, $30,152-158$ (1977).

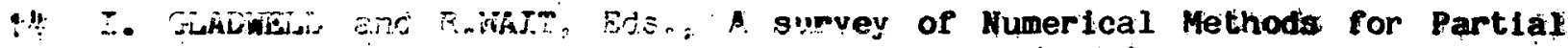
Different lal Equations, Clarendon Press, Oxford (1979). 
15 J.P. HENNART, A general approach to nodal schemes in numerical transport theory, Com. Técnicas, Serie Naranja : No 382, 24p., I IMAS-UNAM (1985).

16 J.P. HENNART, Nodal schemes, mixed-hybrid finite elements and block-centered finite differences, Rapport de Recherche $N^{\circ} 386,59$ p.. INRIA (1985).

17 J.P. HENNART, A general family of nodal schemes, SIAM J. Sci. Stat. Comput., $\underline{3}, 264-287$ (1986).

18 J.P. HENNART, A general finite element framework for nodal methods, MAFELAP 1984, pp. 309-316, J.R. Whiteman, Ed., Academic Press, London (1986).

19 S. LANGENBUCH, W. MAURER, and W. WERNER, Coarse-mesh flux-expansion method for the analys is of space-time effects in large light water reactors cores, Nucl. Sci. Engng., 63, 437-456 (1977).

20 S. LANGENBUCH, W. MAURER and W. WERNER, High-order schemes for neutron kinectics calculations, based on a local polynonial approxination, Nucl. Sci. Engng., 64 , 508-516 (1977).

21 P. LESAINT, On the confergence of Wilson's nonconforming element for solving the elastic problem, Comput. Methods Appl. Mech. Engrg. I, 1-16 $(1976)$.

22 J.C. NEDELEC, Mixed finite elements in $\mathbb{R}^{3}$, Numer. Math., 35, 315-341 (1980). 23 J. C. NEDELEC, A new family of mixed finite element in $\mathbb{R}^{3}$, Numer. Math., to
appear.

24 J.A. NITSCHE, Convergence of nonconforming methods, Mathematical Aspects of Finite Elenents in Partial Differential Equations (C. de Boor, Editor), Academic Press New York, 15-53, (1974).

25 P.A. RAVIART and J.M. THOMAS, A mixed finite element method for second order elliptic problems, in Mathematical Aspects of the Finite Elenent Methods, Lecture Notes in Mathematics 606, pp. 292-315, I. Galligani and E. Magenes, Eds, Springer-Verlag (1977).

26 R.A. SHOBER, R.N. SIMS, and A.F. HENRY, Two nodal methods for solving time-dependent group diffusion equations, Nucl. Sci. Engng., 64, 582-592 (1977).

27 G. STRang, G.J. FIX, An Analysis of the Finite Element Method, Prentice Hall, Englewood Cliffs, New Jersey ( 1973 ).

28 M.R. HAGNER and K. KOEBKE, Progress in nodal reattor analysis, Advances in Reactor Computations, Vol. 2, pp. 941-962, American Nuclear Society. Salt Lake City, Utah (1983). 

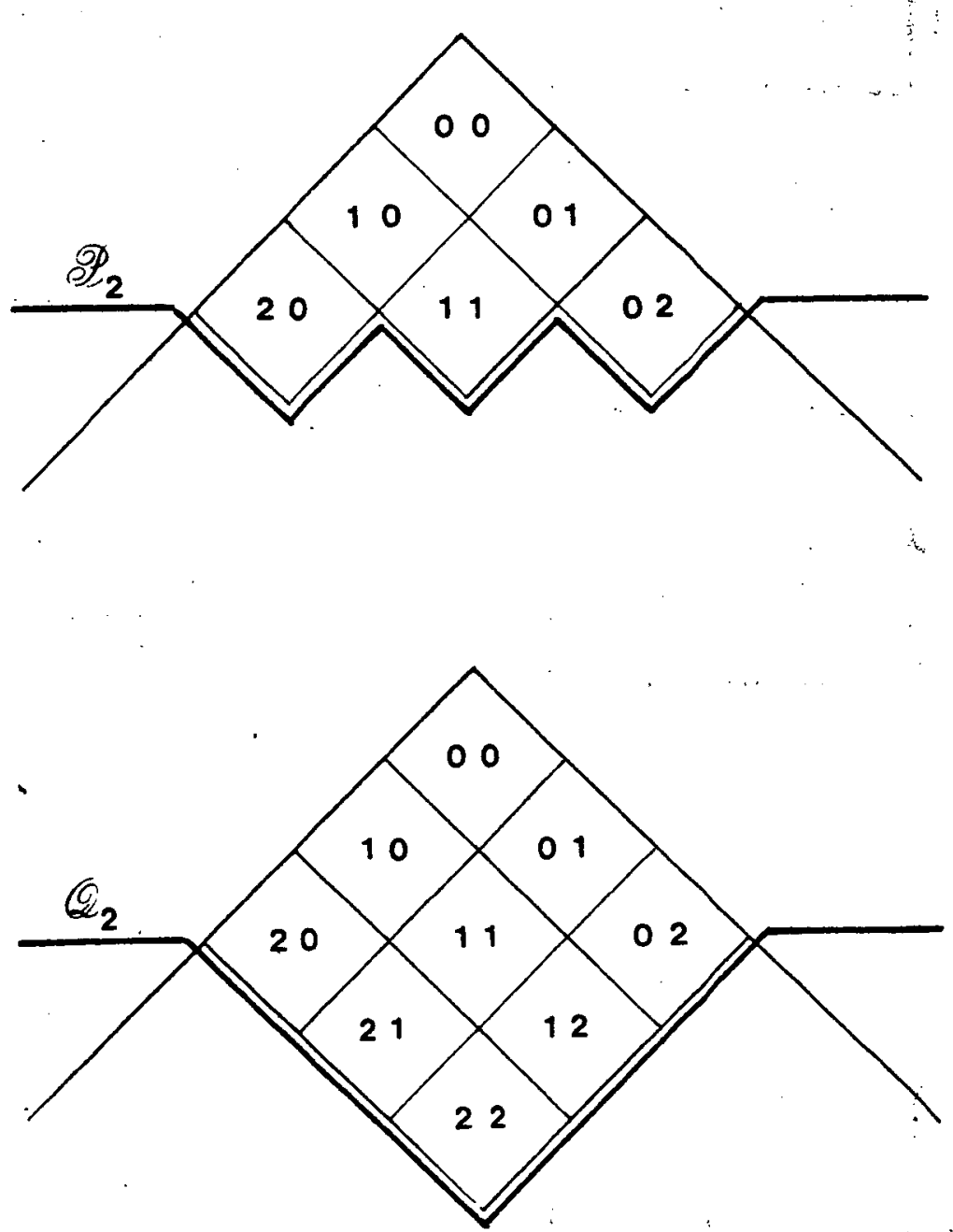

Figure 1: The Pascal triangle and the basic polynomial spaces $\mathscr{P}_{2}$ and $\mathscr{Q}_{2}$ 

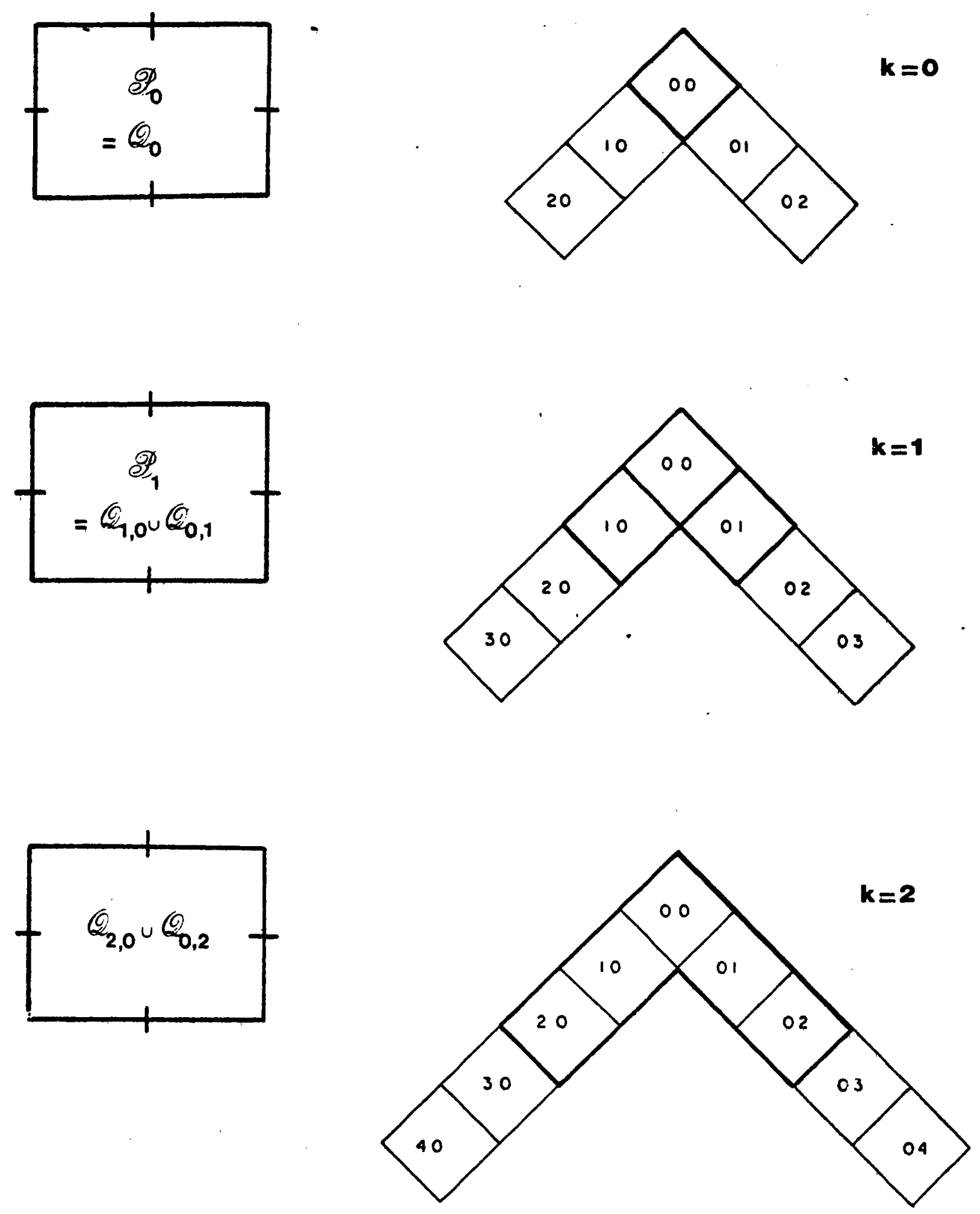

Figure 2 : The Langenbuch-Maurer-Werner I or sum nodal schemes. 

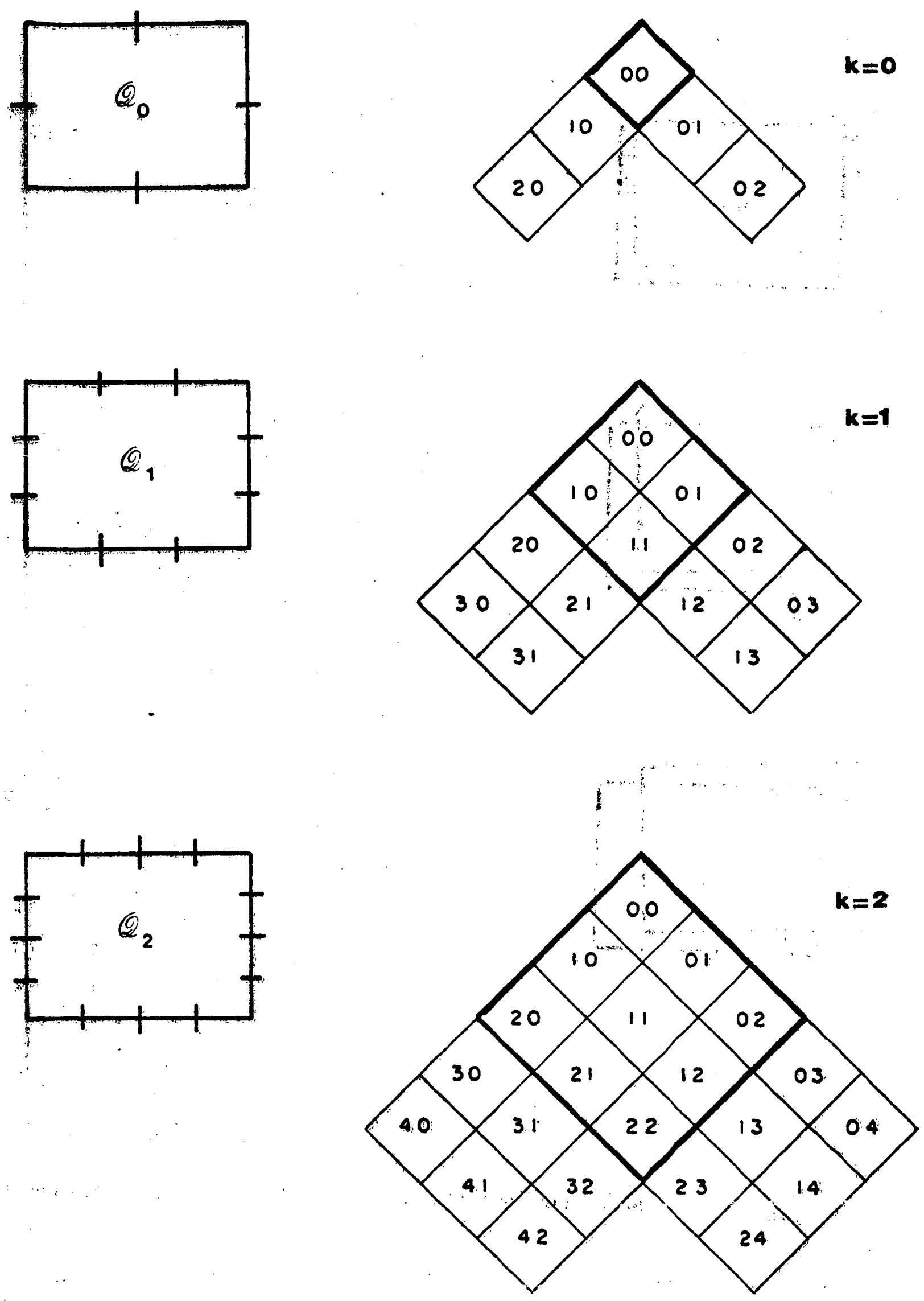

Figure: 3: The Hennart nodal schemes in $2 D$. 

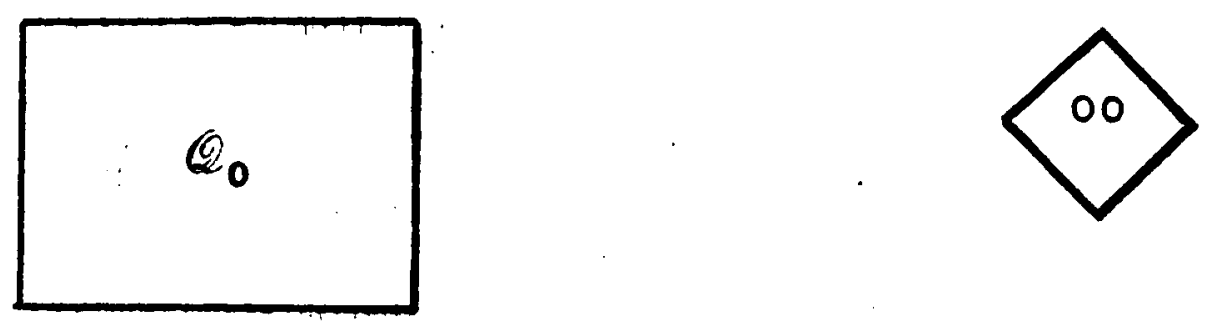

$$
\mathbf{k}=\mathbf{0}
$$

(n)
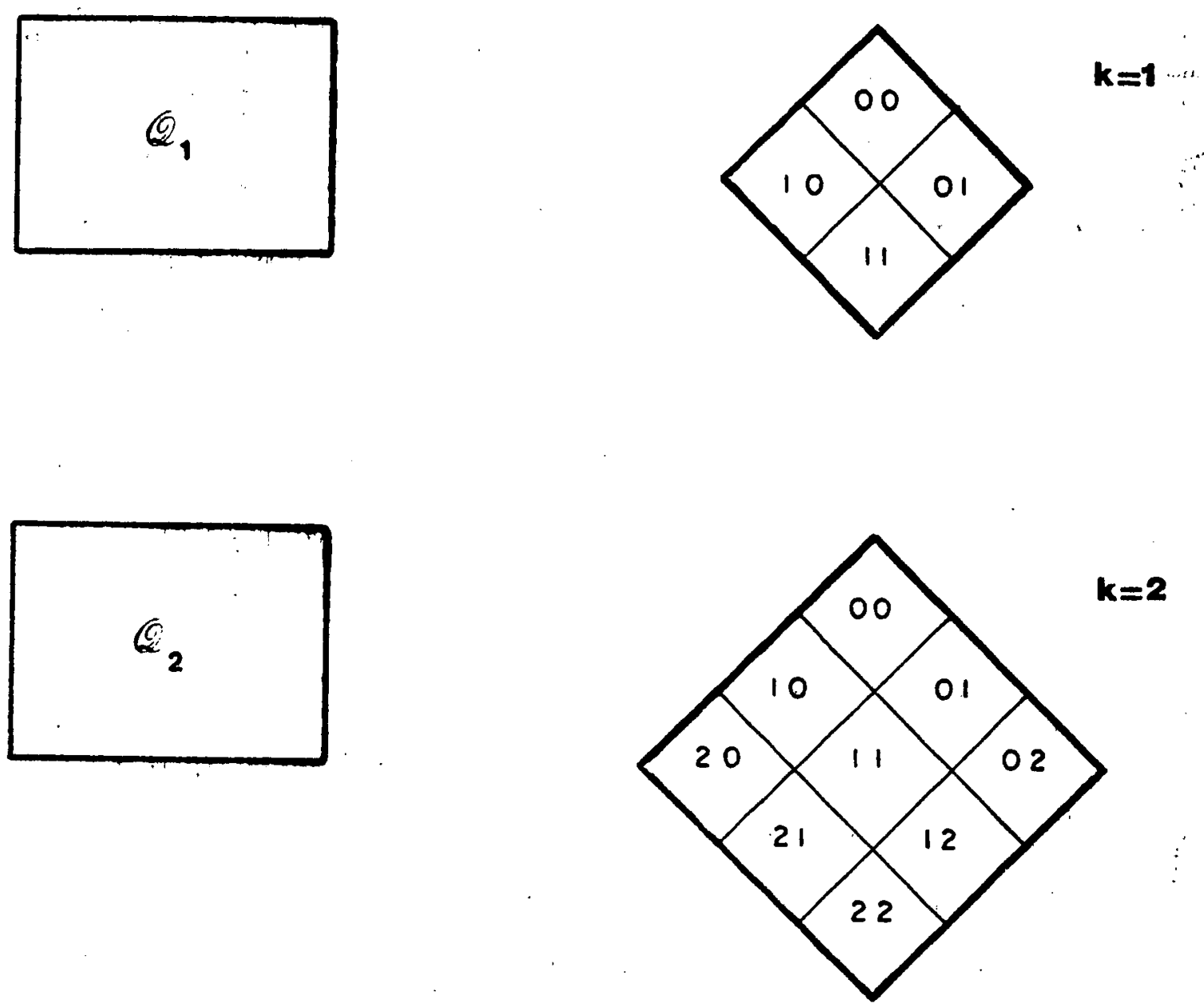

Figure 4: The scalar varlable for the Raviart-Thomas mixed schemes. 

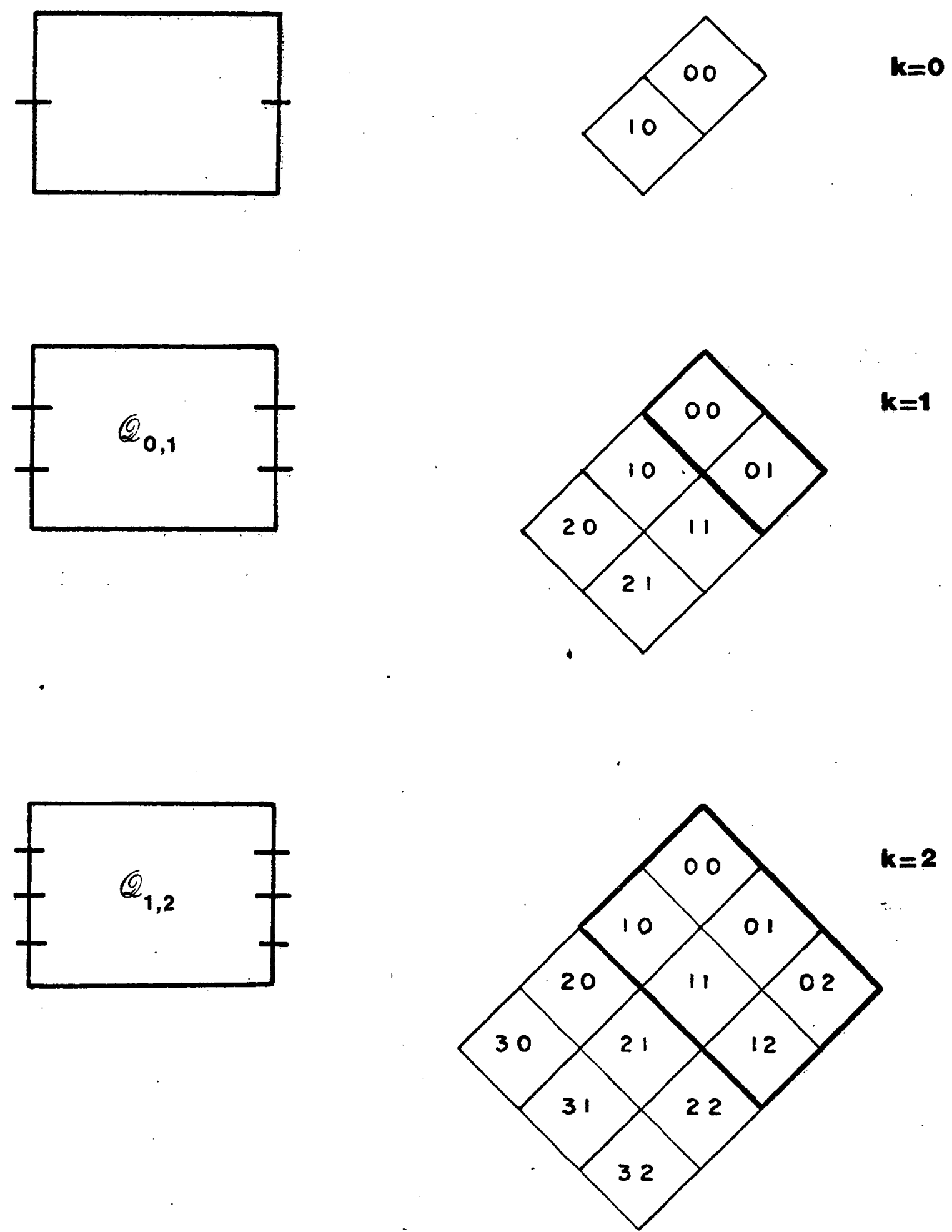

Figure 5: The first Component $v_{1}$ of the vector variable for the Raviart-Thomas mixed schemes. 

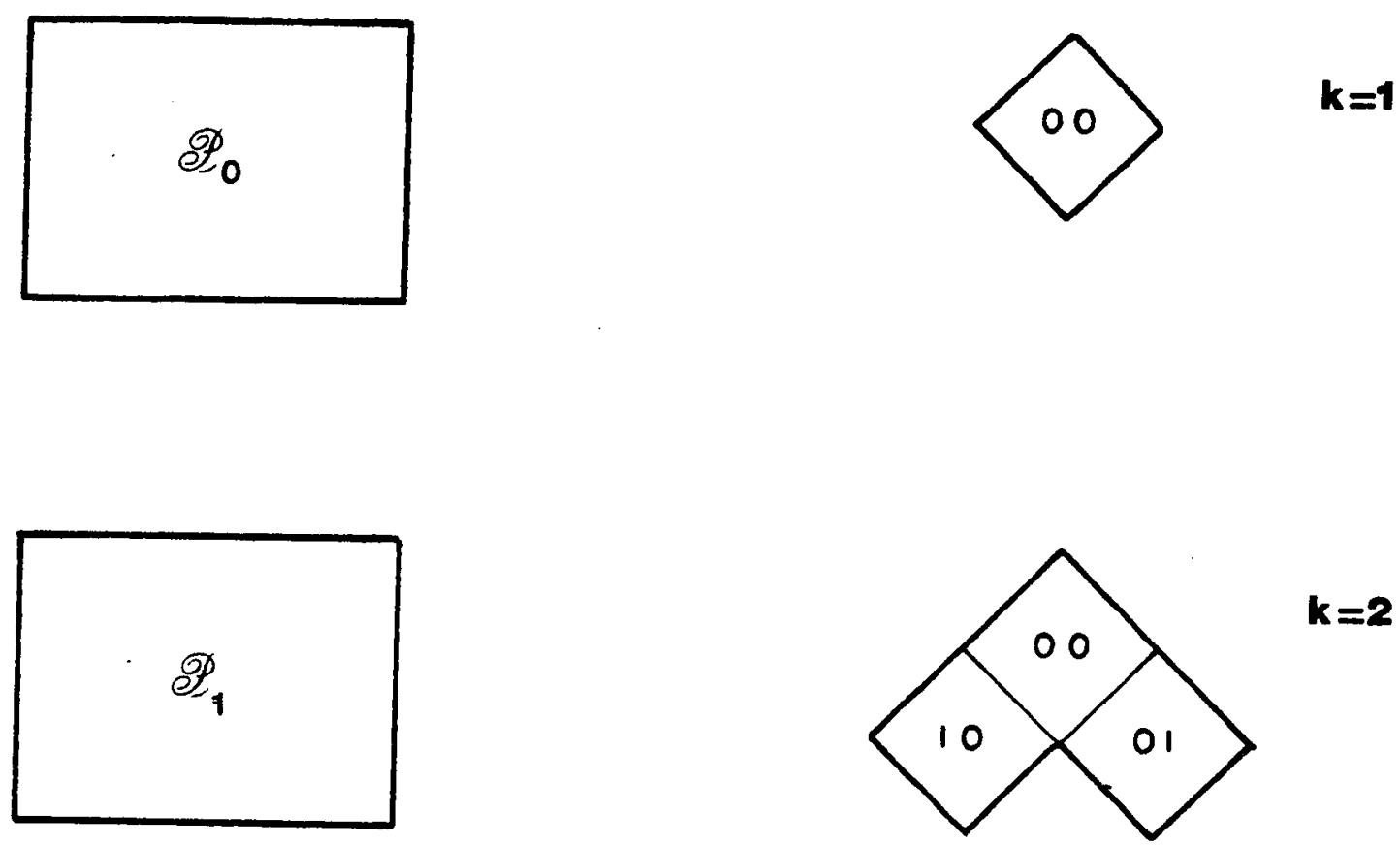

Figure 5 : The scalar variable for the Brezzi-Luagles-Marini mised schemes. 

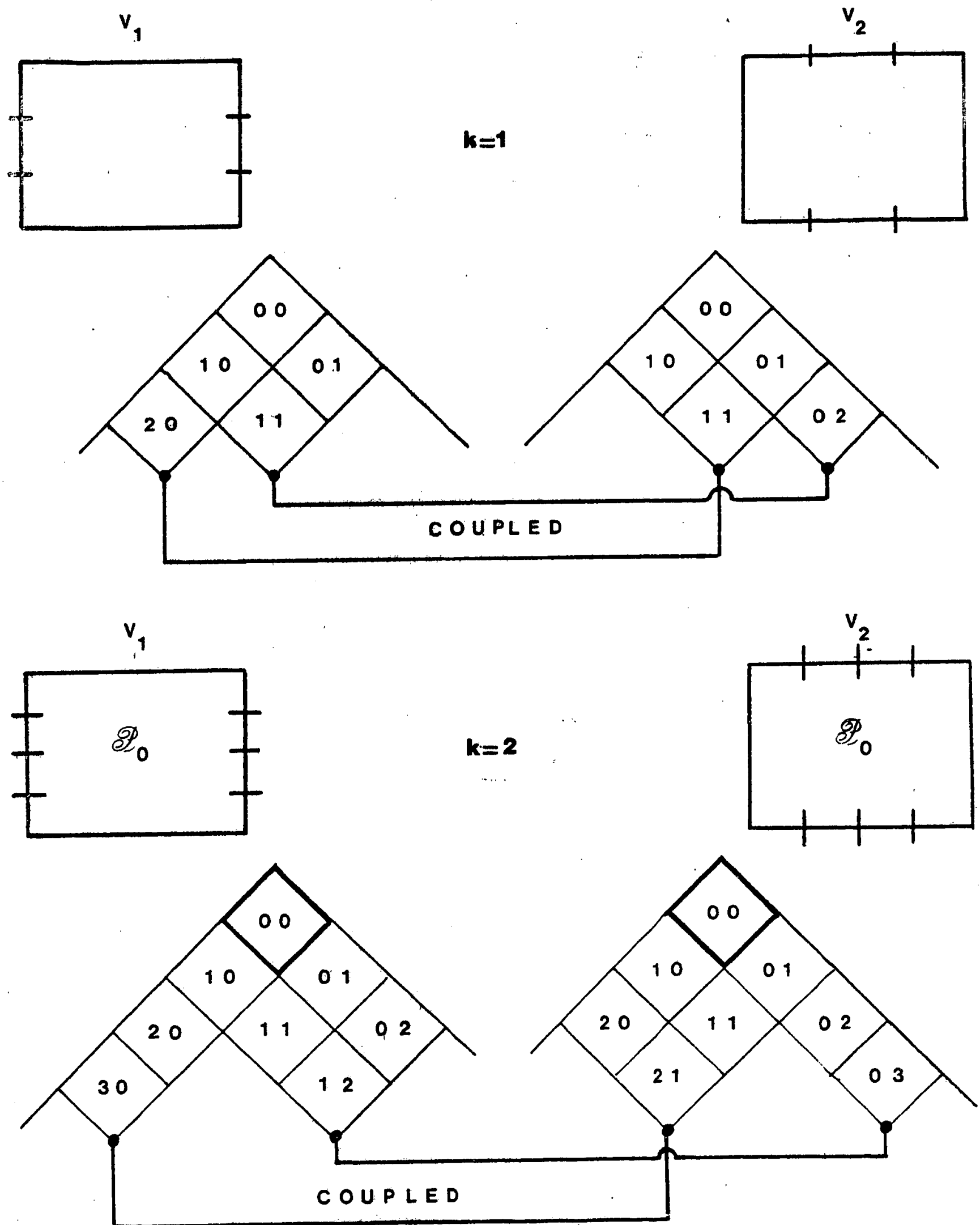

Figure 1 : The vector varlables for the Brezzi-Douglas-Marini mixed schemes. 

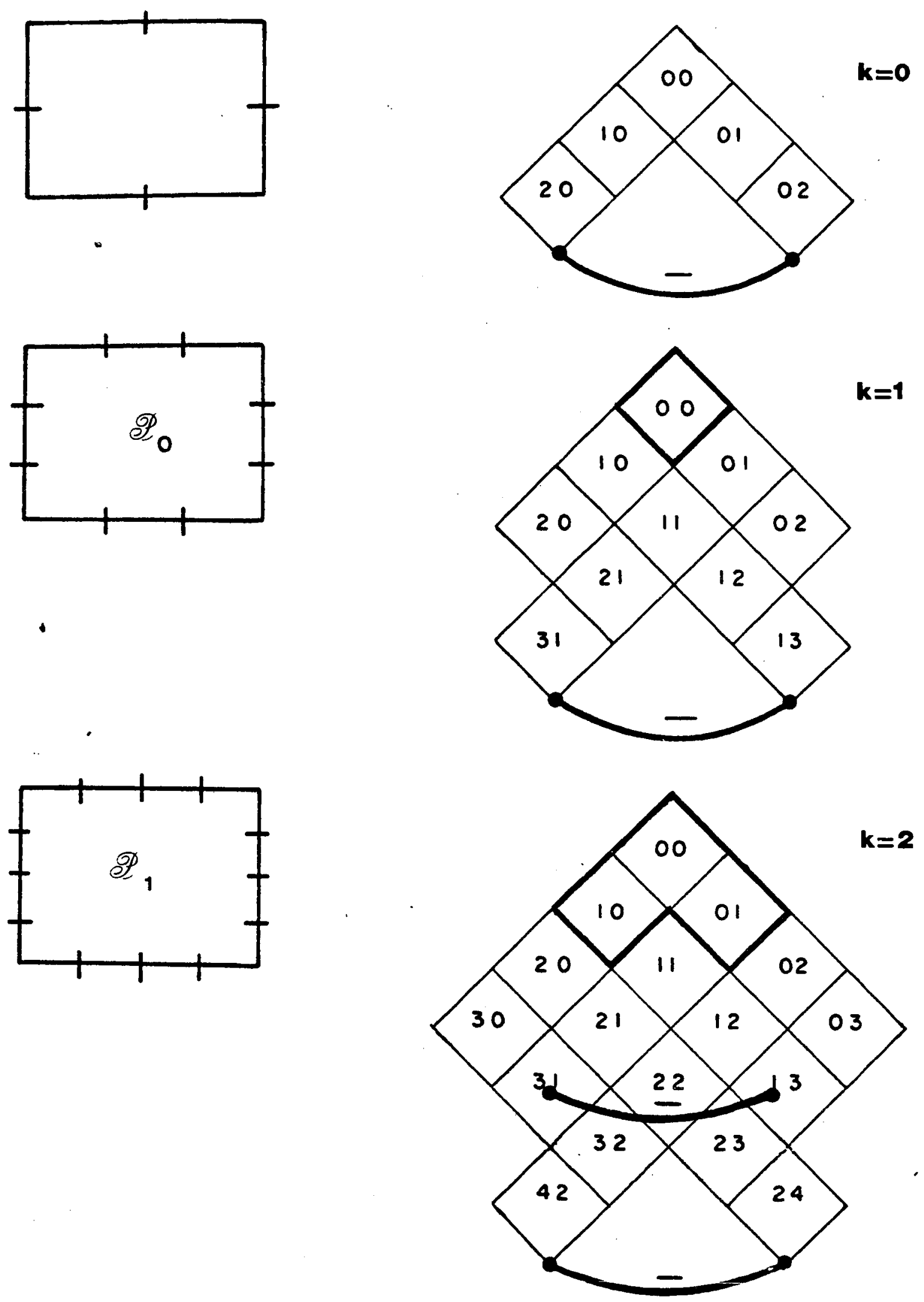

Figure 8 : Nodal finite elements of the BDM type in 2D. 

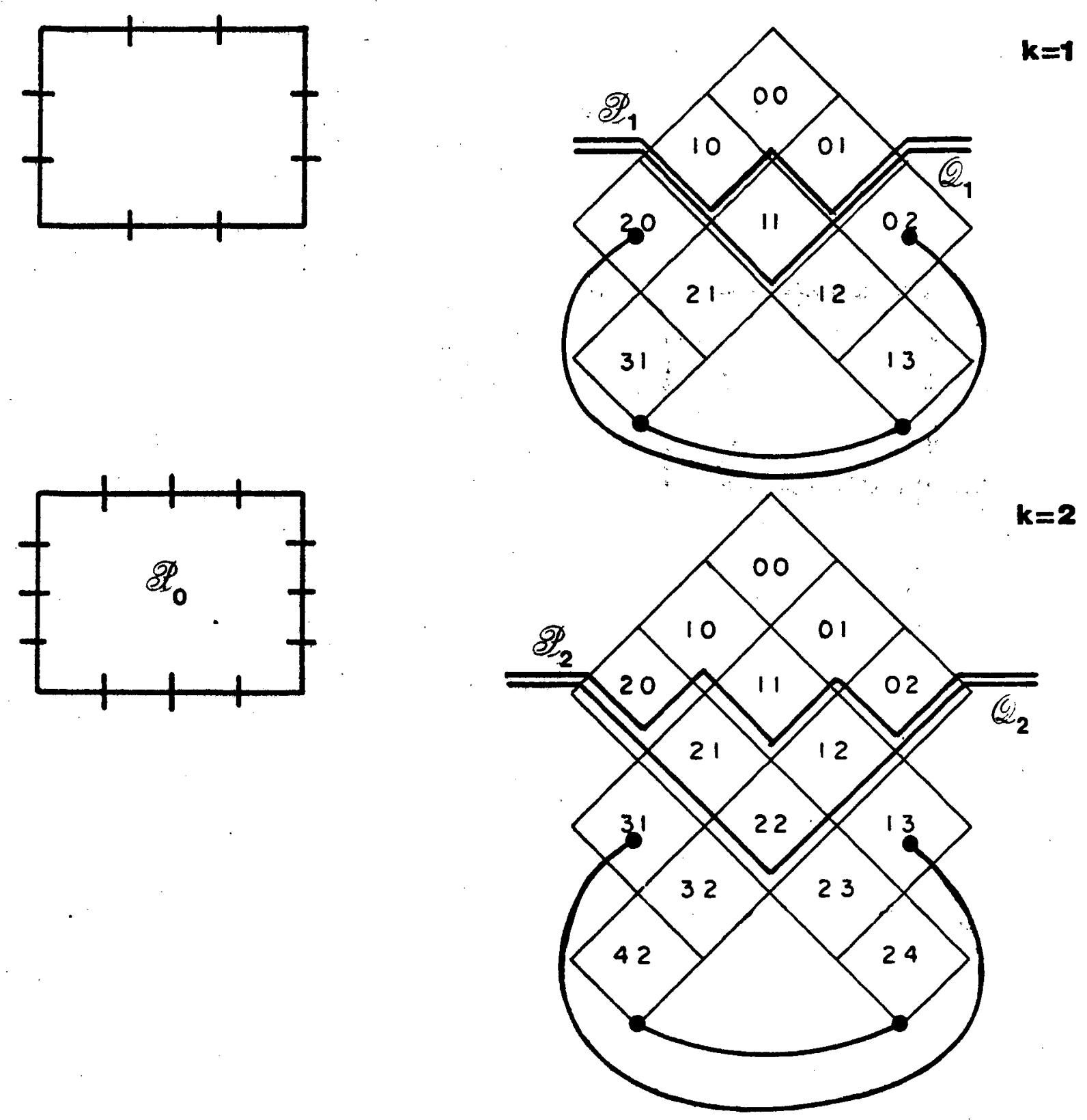

Flgure 9: Family a : a family of nodal finite elements not climbing correctly in order. 

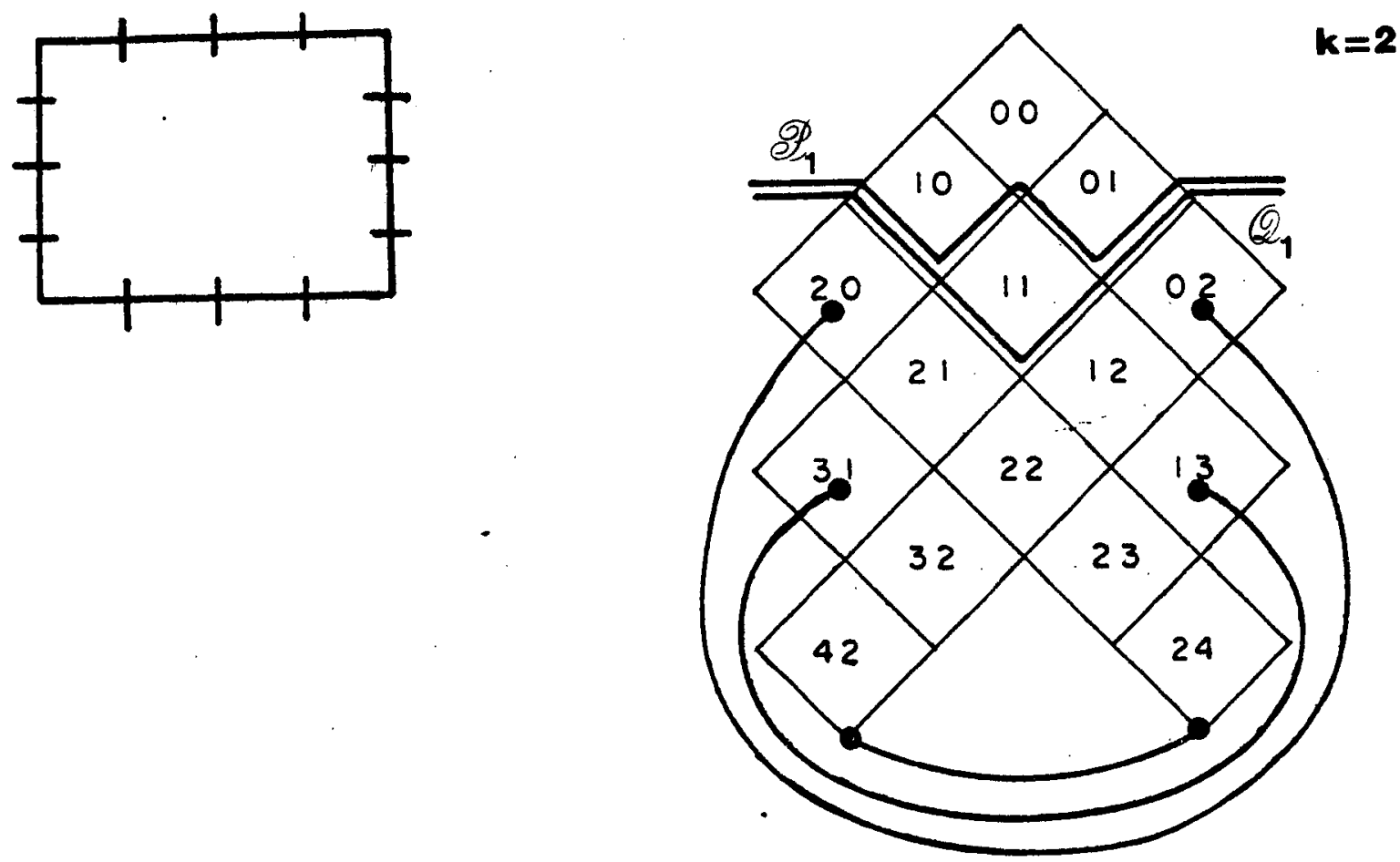

Figure 10 : Family b a family of nodal finite elements not climbing correctiy in order. 

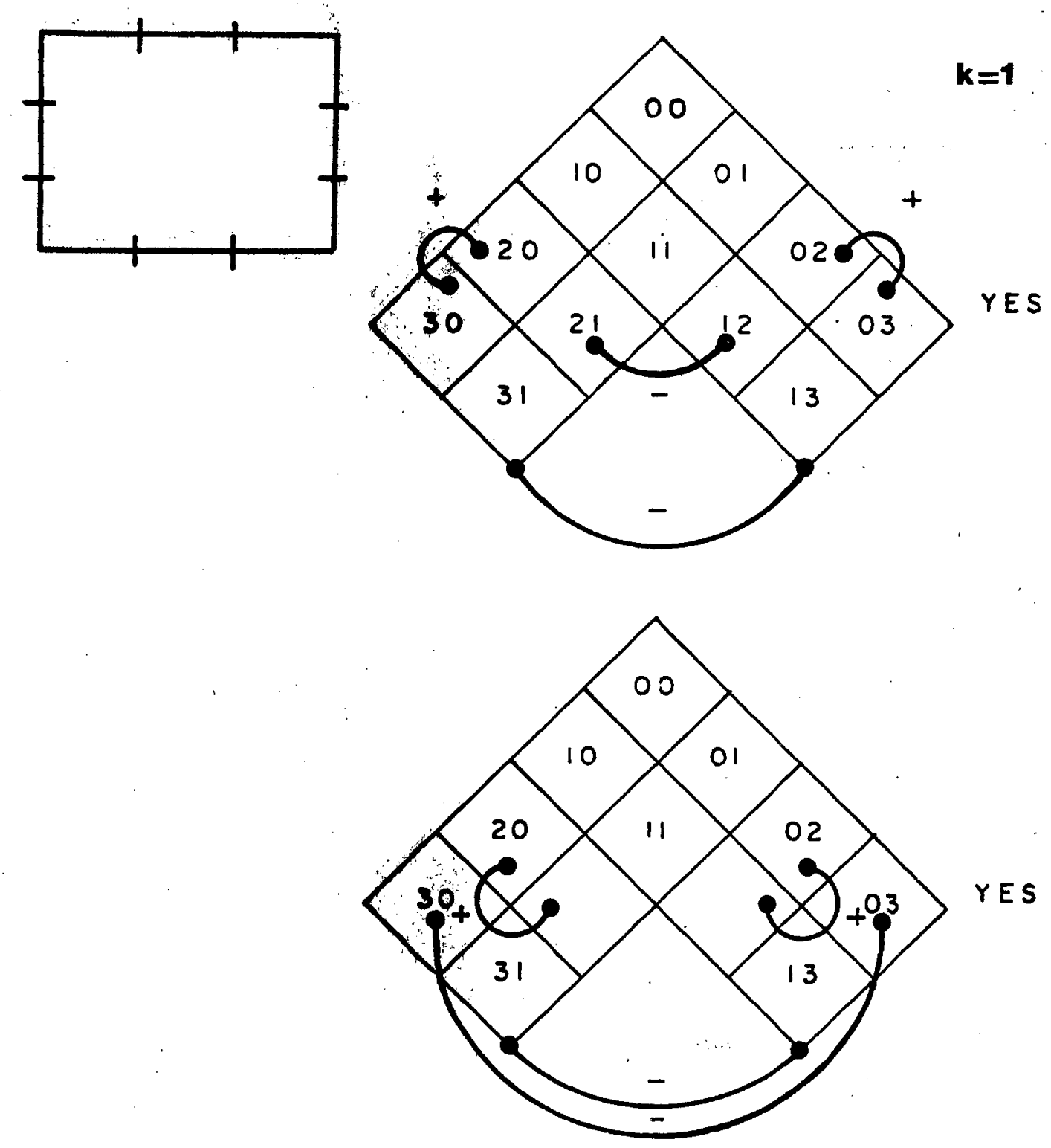

Figure 11 : Nodal finite element schemes resulting from a modification of Algor Ithm 1 for family a $-k=1$. 

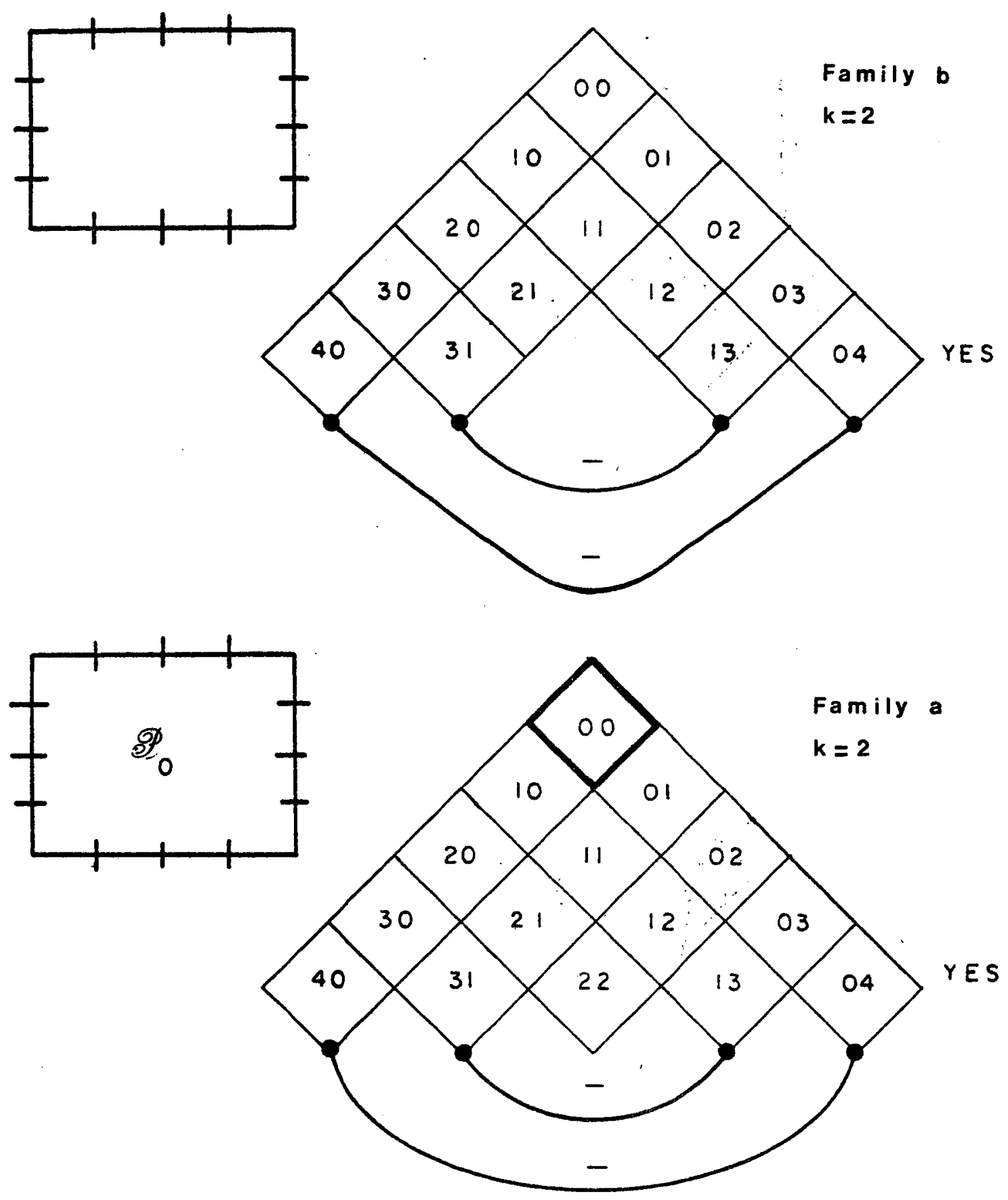

Figure 12 : Nodal finite element schemes resuliting frow a modification of Algorithm 1 for families $a$ and $b-k=2$. 

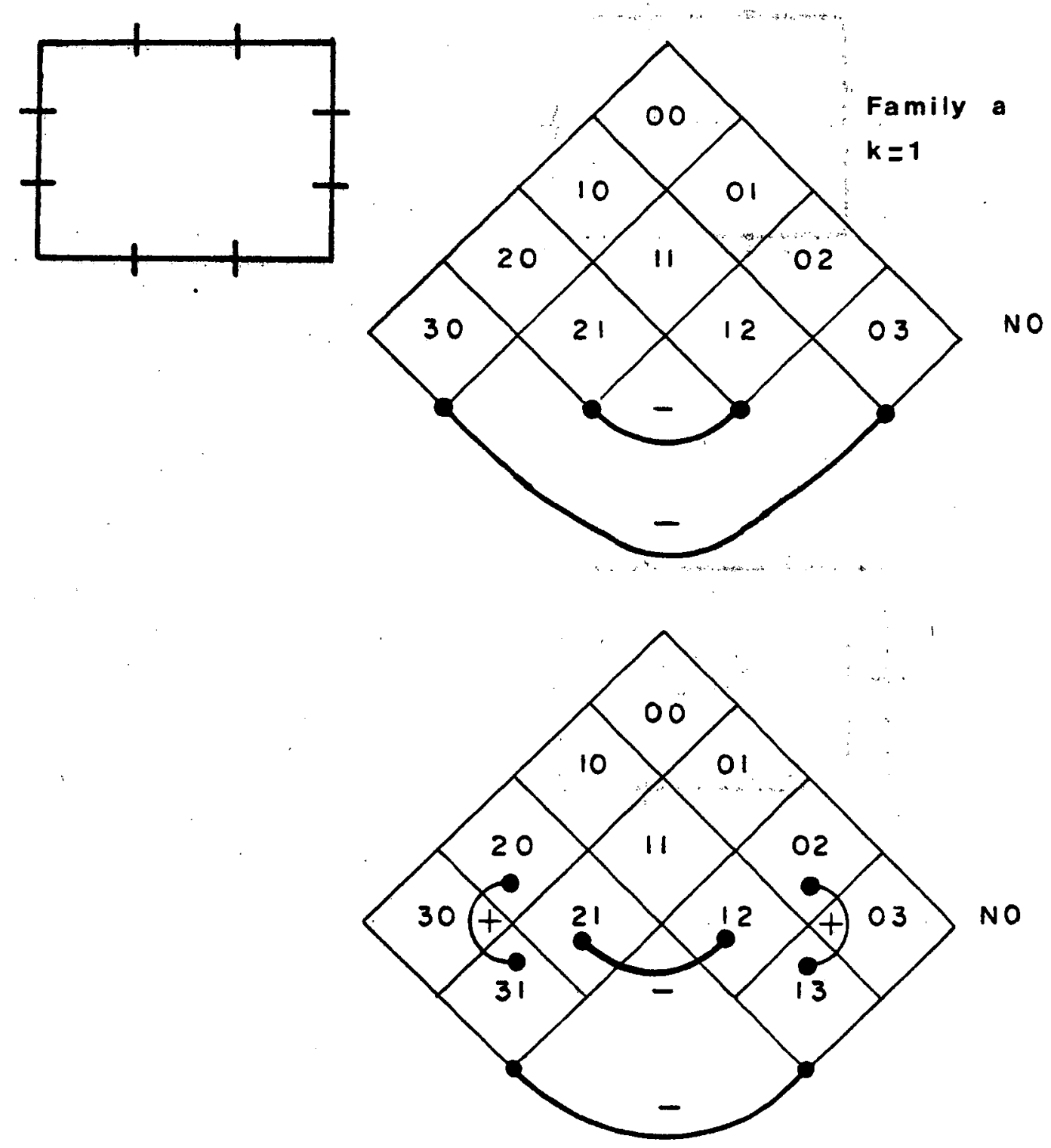

Figure 13: Schemes resulting from a modification of Algorithm 1 which are not unisolvent (Family $\#, k=1$ ). 

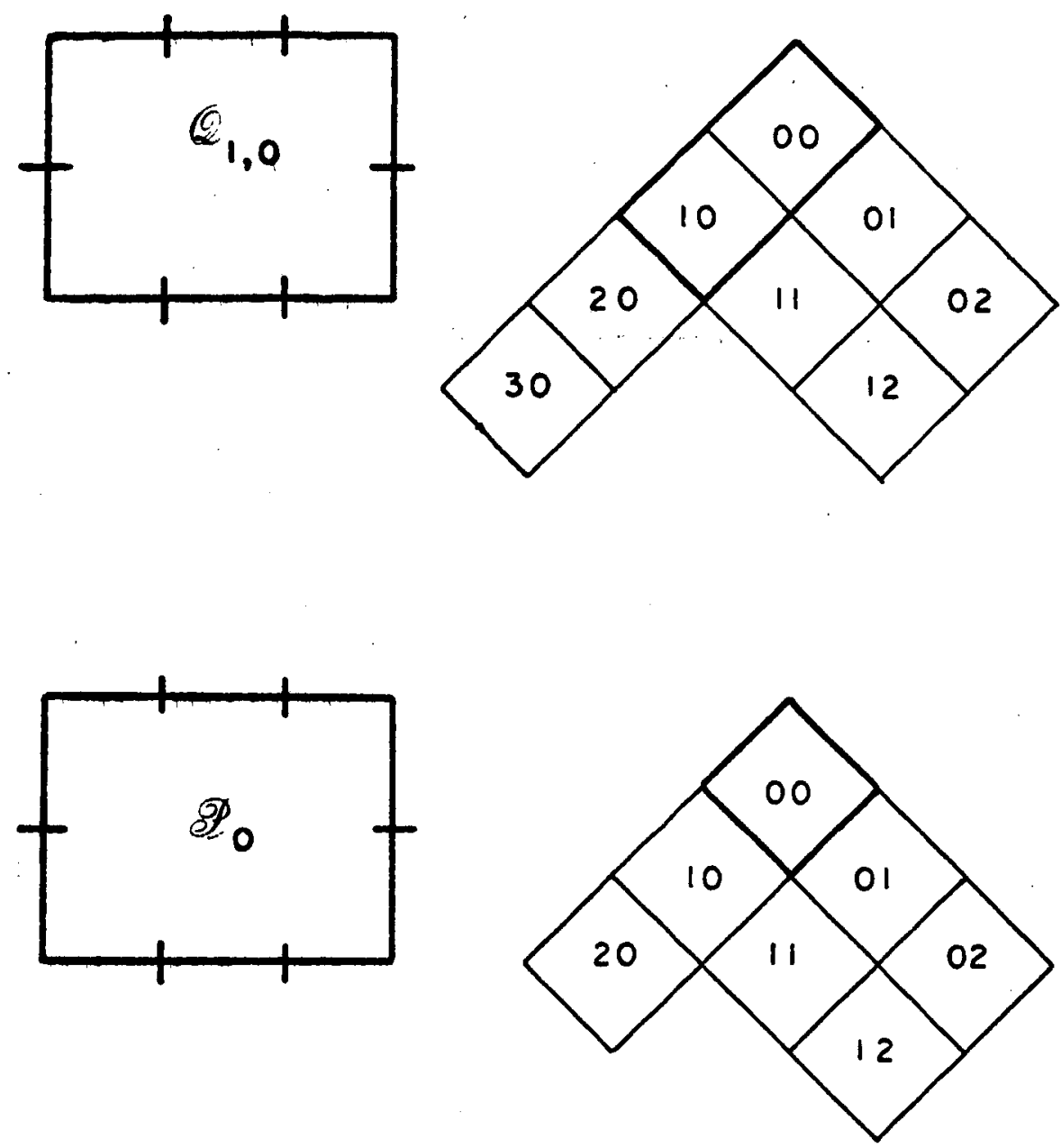

Flgure 14: Examples of asymmetric nodal finite elements. 


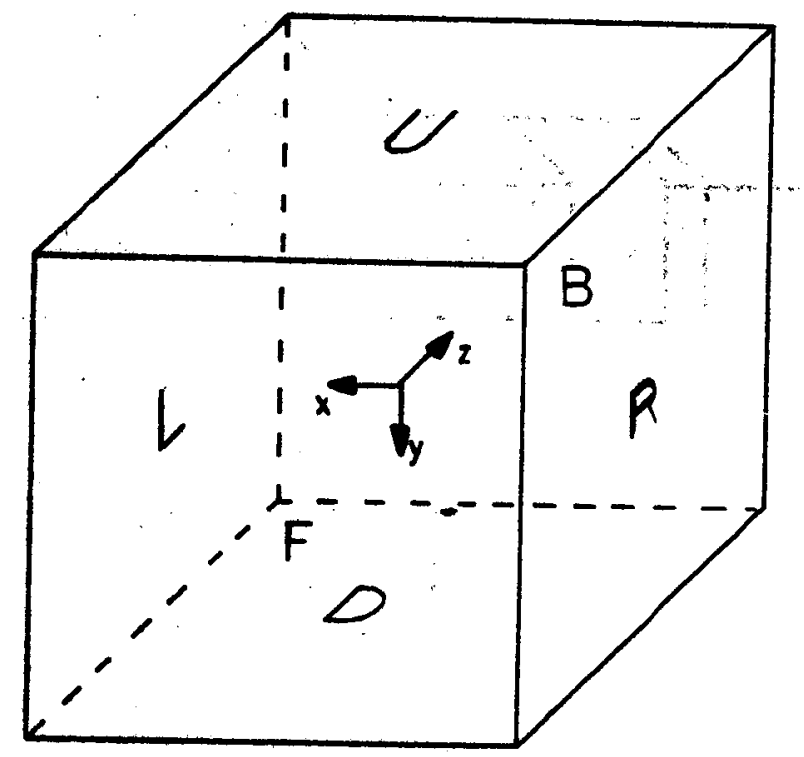

Figure 15 : Notation for the three-dimensional case. 


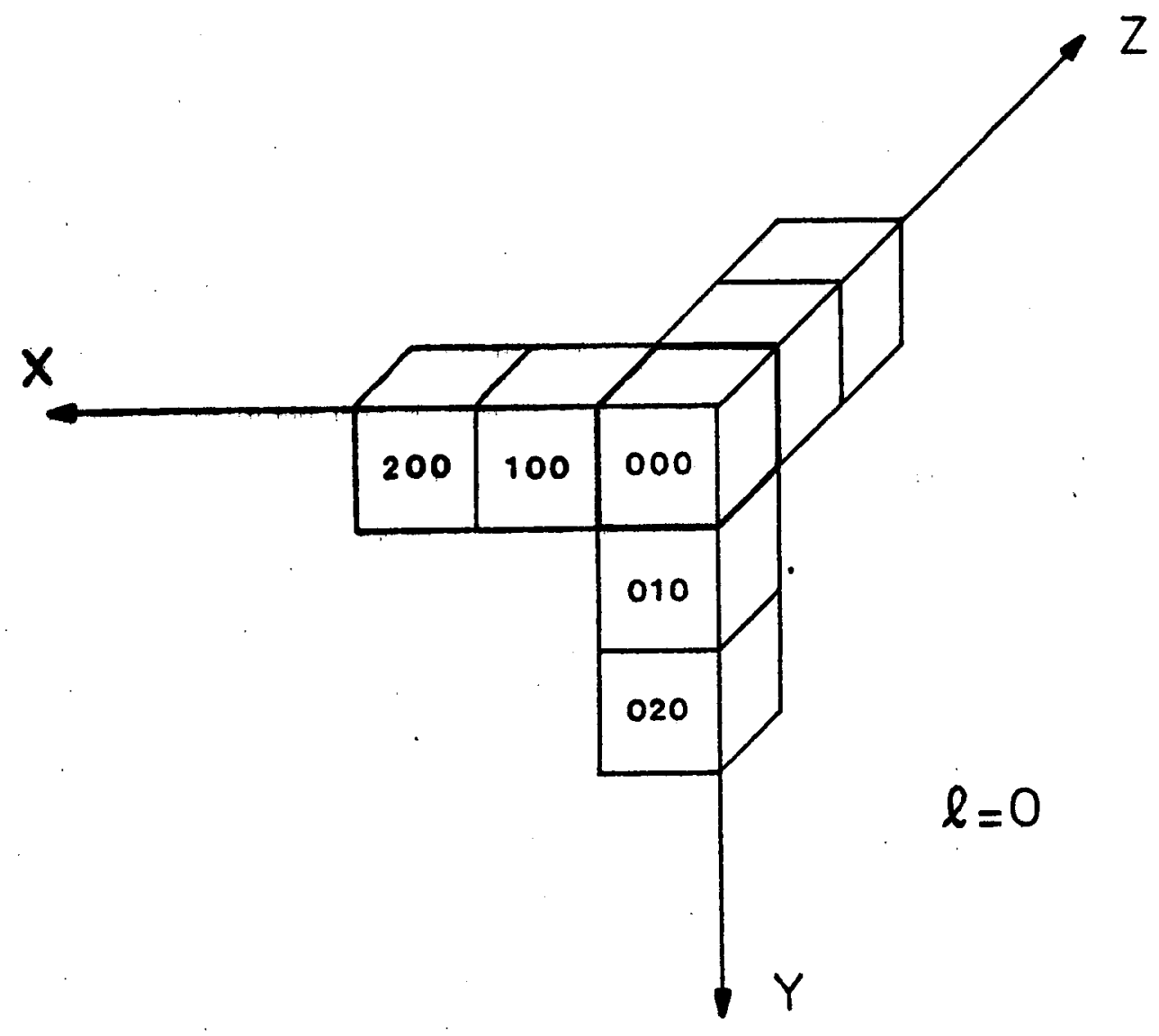

Plgure 16: Hennart nodal scheme in 3D $-l=0$. 


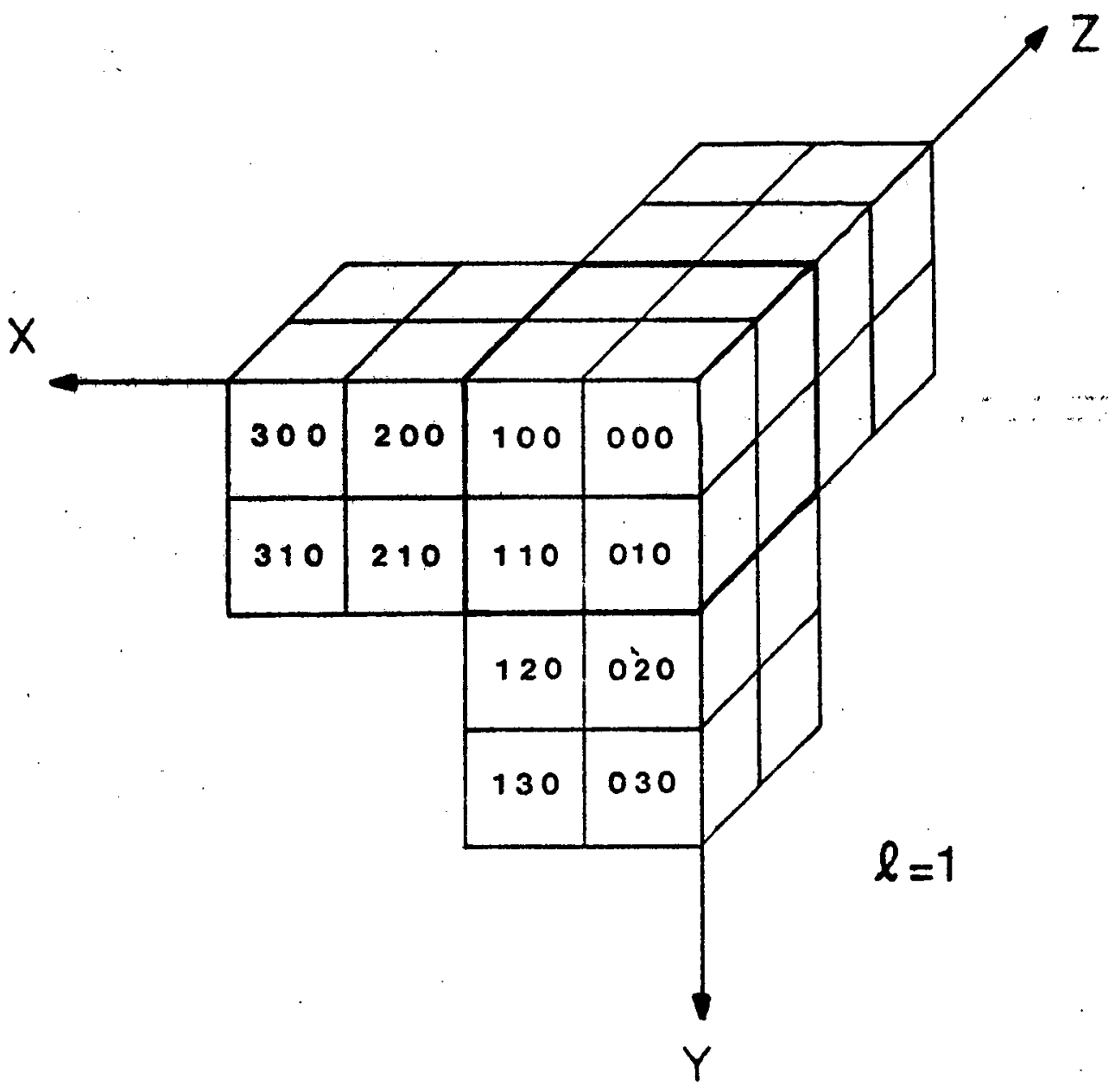

F1gure 17 : Hennart nodal scheme in 3D $-\ell=1$. 


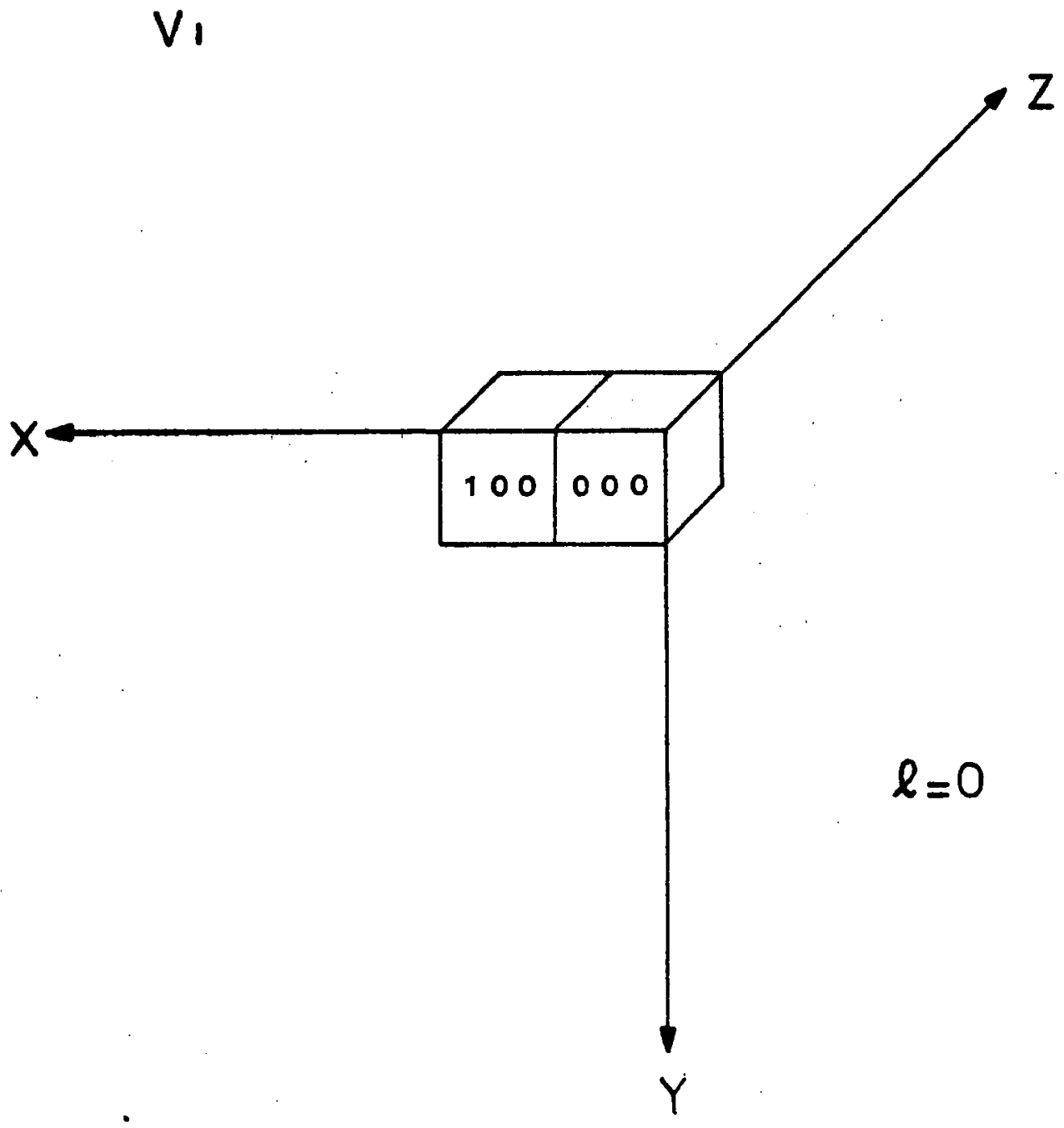

F1sure 18 : Original Nédélec mixed finite elements in 3D. First component of vector variábie $-\ell=0$. 


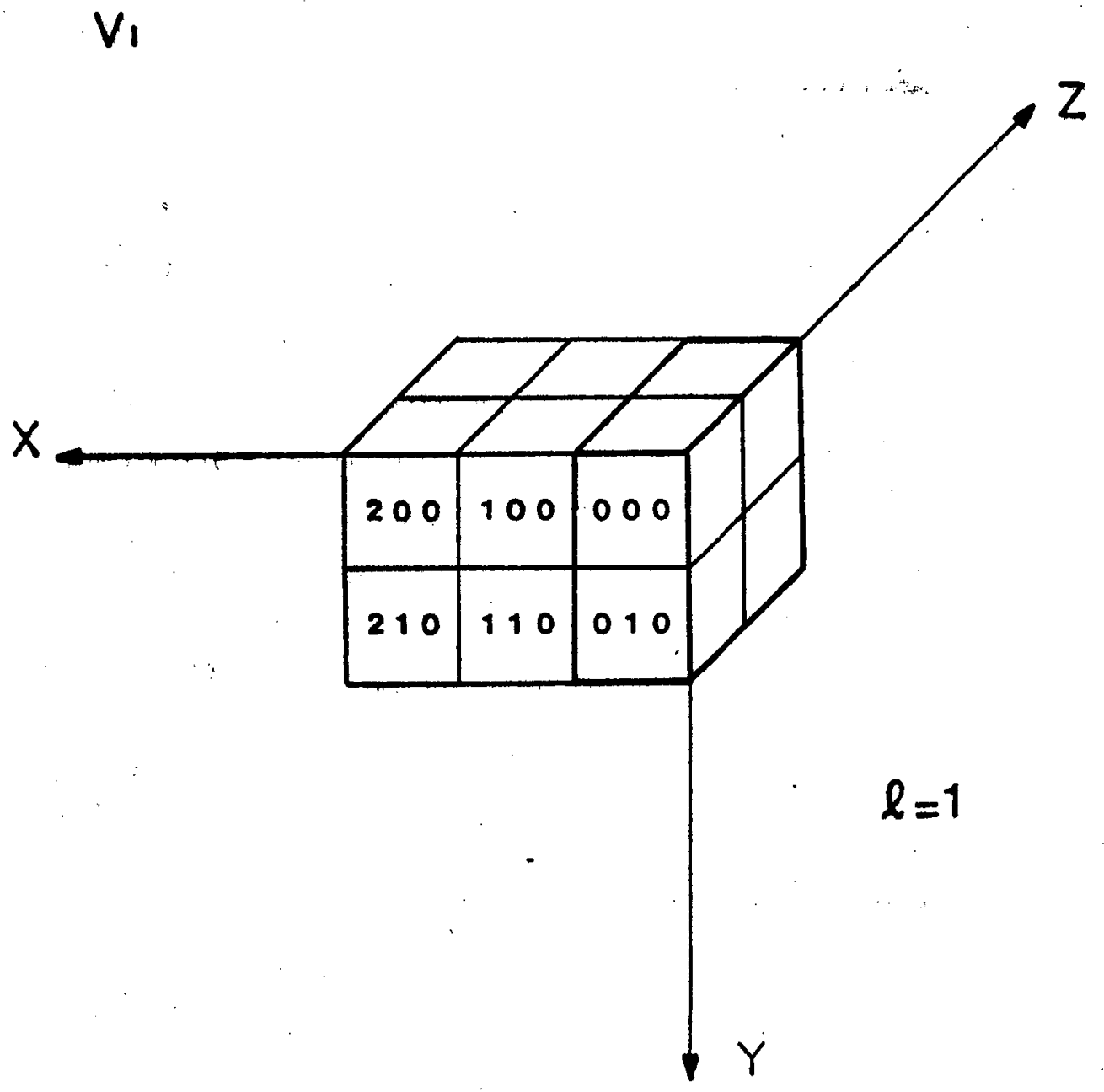

Flgure 19: Orlginal Nédélec mixed finite elements in 3j. First couponent of vector variable $-\imath=1$. 


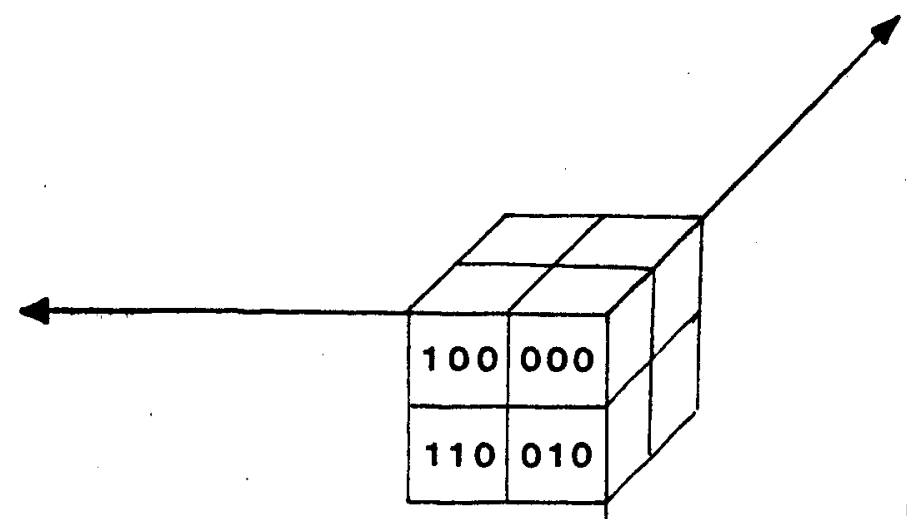

$\ell=1$

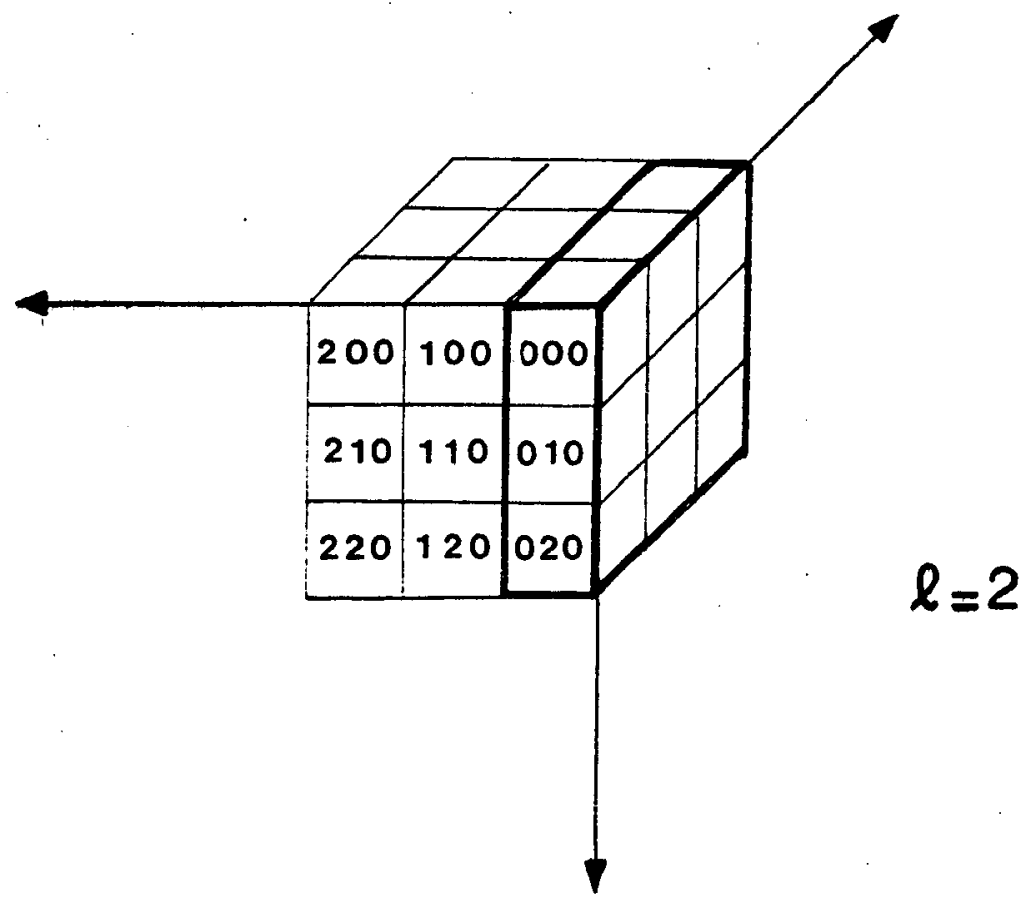

Figure 20: Mew Médélec mixed finite elements in 3D. First component of vector variable $-\ell=1$ and $\ell=2$. 


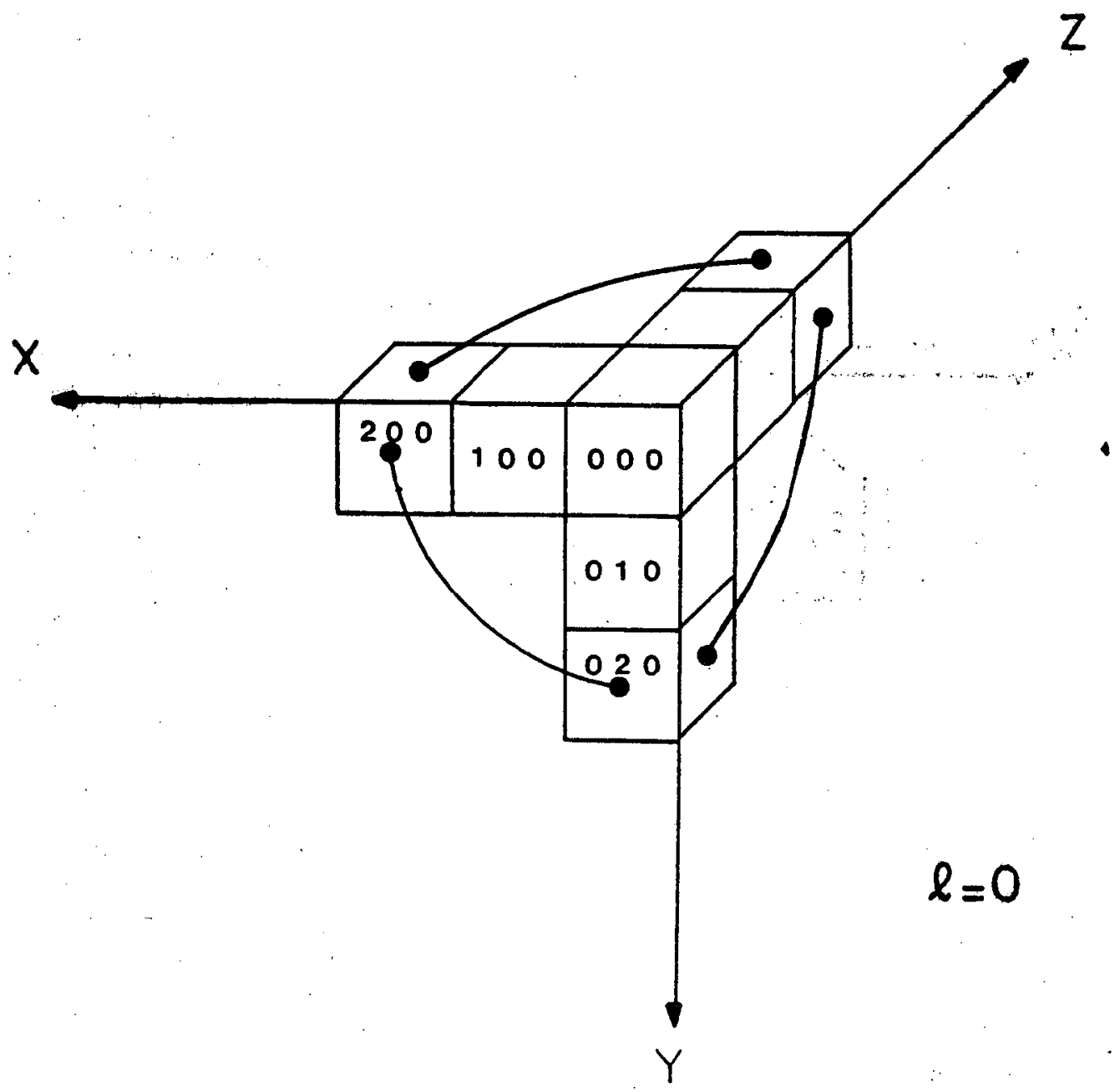

Flaure 21 ; Nodal finite elements of BDM type in 3D $-\ell=0$. 


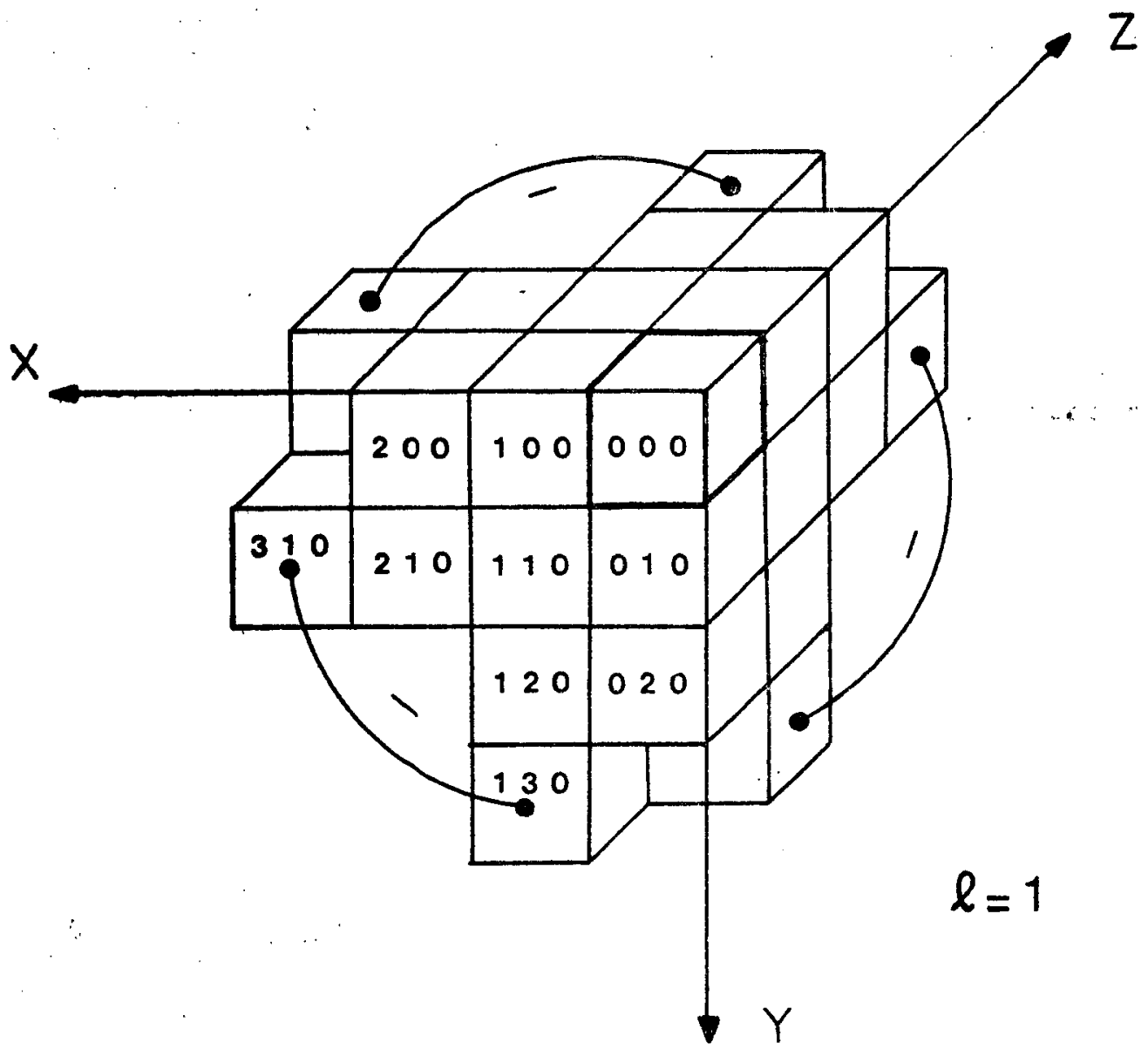

Figure 22 : Nodal finite elements of BDM type in 3D $-\ell=1$.

\author{
Imprimé en France \\ par
}

PInstitut National de Recherche en Informatique et en Automatique 
